\title{
Inducible Activation of ERK5 MAP Kinase Enhances Adult Neurogenesis in the Olfactory Bulb and Improves Olfactory Function
}

\author{
Wenbin Wang, ${ }^{1}$ Song Lu, ${ }^{1}$ Tan Li, ${ }^{1,2}$ Yung-Wei Pan, ${ }^{3}$ Junhui Zou, ${ }^{1}$ Glen M. Abel, ${ }^{1}$ Lihong Xu, ${ }^{2}$ Daniel R. Storm, ${ }^{4}$ \\ and Zhengui Xia ${ }^{1,3}$ \\ ${ }^{1}$ Toxicology Program, Department of Environmental and Occupational Health Sciences, University of Washington, Seattle, Washington 98195, ${ }^{2}$ Department \\ of Biochemistry and Genetics, Zhejiang University, Hangzhou, Zhejiang 310058, China, ${ }^{3}$ Graduate Program in Molecular and Cellular Biology, and \\ ${ }^{4}$ Department of Pharmacology, University of Washington, Seattle, Washington 98195
}

Recent discoveries have suggested that adult neurogenesis in the subventricular zone (SVZ) and olfactory bulb (OB) may be required for at least some forms of olfactory behavior in mice. However, it is unclear whether conditional and selective enhancement of adult neurogenesis by genetic approaches is sufficient to improve olfactory function under physiological conditions or after injury. Furthermore, specific signaling mechanisms regulating adult neurogenesis in the SVZ/OB are not fully defined. We previously reported that ERK5, a MAP kinase selectively expressed in the neurogenic regions of the adult brain, plays a critical role in adult neurogenesis in the SVZ/OB. Using a site-specific knock-in mouse model, we report here that inducible and targeted activation of the endogenous ERK5 in adult neural stem/progenitor cells enhances adult neurogenesis in the OB by increasing cell survival and neuronal differentiation. This conditional ERK5 activation also improves short-term olfactory memory and odor-cued associative olfactory learning under normal physiological conditions. Furthermore, these mice show enhanced recovery of olfactory function and have more adult-born neurons after a zinc sulfate-induced lesion of the main olfactory epithelium. We conclude that ERK5 MAP kinase is an important endogenous signaling pathway regulating adult neurogenesis in the SVZ/OB, and that conditional activation of endogenous ERK5 is sufficient to enhance adult neurogenesis in the $\mathrm{OB}$ thereby improving olfactory function both under normal conditions and after injury.

Key words: adult neurogenesis; ERK5; MAP kinase; olfaction

\section{Introduction}

In the adult mammalian brain, the subventricular zone (SVZ) along the lateral ventricle harbors steadily dividing neuronal precursors that migrate along the rostral migratory stream (RMS) to the olfactory bulb $(\mathrm{OB})$ where they differentiate into local inhibitory interneurons (Whitman and Greer, 2009). Several recent discoveries suggest that adult olfactory neurogenesis may affect some forms of olfactory behavior in mice (Kageyama et al., 2012). For example, Moreno et al., (2009) showed that the odor enrichment-improved odor discrimination is blocked by infusion of the anti-mitotic drug cytosine- $\beta$-D-arabinofuranoside (AraC). Breton-Provencher and Saghatelyan (2012) reported that AraC infusion reduced odor-detection sensitivity and short-

\footnotetext{
Received Sept. 8, 2014; revised April 13, 2015; accepted April 15, 2015.

Author contributions: W.W., L.X., D.R.S., and Z.X. designed research; W.W., S.L., T.L., Y.-W.P., J.Z., and G.M.A. performed research; W.W., S.L., T.L., J.Z., and Z.X. analyzed data; W.W., S.L., D.R.S., and Z.X. wrote the paper.

This work was supported by the National Institutes of Health Grants R01 MH95840 to Z.X. and R01 DC004156 to D.R.S. We thank Dr Chay T. Kuo, Duke University Medical Center, for providing the Nestin-CreER ${ }^{\mathrm{TM}}$ mice, and members of the Xia laboratory for critical reading of the paper.

The authors declare no competing financial interests.

Correspondence should be addressed to Dr Zhengui Xia, Toxicology Program in the Department of Environmental and Occupational Health Sciences, Box 357234, University of Washington, Seattle, WA 98195. E-mail: zxia@u.washington.edu.

DOI:10.1523/JNEUROSCI.3745-14.2015

Copyright $\odot 2015$ the authors $\quad 0270-6474 / 15 / 357833-17 \$ 15.00 / 0$
}

term olfactory memory in mice without affecting long-term odor-associative memory. In another study, Ara-C treatment impaired long-term olfactory memory retention, although it did not alter basic olfactory function. (Sultan et al., 2010).

ERK5, a member of the MAP kinase family that also includes ERK1/2, JNK, and p38MAPK, was recently identified as an endogenous signaling pathway that regulates adult neurogenesis (Pan et al., 2013b). ERK5 is specifically phosphorylated and activated by MEK5 (English et al., 1995; Zhou et al., 1995), and MEK5 is specific for ERK5 with ERK5 as its sole identified substrate (English et al., 1995; Zhou et al., 1995). We recently reported that ERK5 is specifically expressed in two neurogenic regions in the adult brain, including the SVZ-RMS-OB axis (Pan et al., 2012a,b; Li et al., 2013b). Furthermore, inducible and targeted deletion of erk5 in the adult neurogenic regions impairs several aspects of adult olfactory neurogenesis, including neuronal differentiation, migration, and survival (Li et al., 2013b). Deletion of erk5 leads to several olfactory behavior deficits, including reduced odor-detection sensitivity and short-term memory for odorants (Pan et al., 2012a). These data provide further evidence that adult neurogenesis is necessary for normal olfactory function.

Because aging, injury, and neurodegenerative diseases including Parkinson's disease, as well as Alzheimer's disease, are associ- 
ated with impaired olfactory function, it is important to determine whether enhancement of adult neurogenesis will improve olfactory memory or function. Here, we used a site-specific constitutively activated MEK5 (caMEK5) knock-in mouse line to conditionally activate endogenous ERK5 specifically in the adult neurogenic regions of the brain (Wang et al., 2014). We report that genetic activation of ERK5 increases adult neurogenesis in the $\mathrm{OB}$ and improves olfactory function.

\section{Materials and Methods}

Animals. The ROSAcaMEK5-eGFP knock-in mouse line was generated in our laboratory as previously described (Wang et al., 2014). The Nestin$\mathrm{CreER}^{\mathrm{TM}}$ transgenic mouse line (Kuo et al., 2006) and the reporter line Rosa26-loxP-stop-loxP-TdTomato (line Ai14; Madisen et al., 2010) were previously described. An inducible and conditional caMEK5 mouse line was generated by crossing the caMEK5-eGFP mice with NestinCreER ${ }^{\mathrm{TM}}$ mice; the resulting Nestin-CreER ${ }^{\mathrm{TM}}$ :caMEK5 mice express caMEK5-eGFP in Nestin-expressing cells from the ROSA26 locus upon tamoxifen administration. Nestin-CreER ${ }^{\mathrm{TM}}$ :caMEK5 mice were also crossed with the reporter line Rosa26-loxP-stop-loxP-TdTomato to obtain Nestin-CreER ${ }^{\mathrm{TM}}$ :caMEK5/TdTomato mice. All animals were in mixed C57BL/6 and SV129 background. Animals were housed under standard conditions ( $12 \mathrm{~h}$ light/dark cycle) with food and water provided ad libitum. Both male and female mice were used in cellular studies, whereas only male mice were used in behavioral studies. Littermate controls were of the same sex within each experiment. All experimental procedures were approved by the University of Washington Institutional Animal Care and Use Committee.

Tamoxifen administration and BrdU labeling. To induce Nestin$\mathrm{CreER}^{\mathrm{TM}}$-mediated recombination of caMEK5-eGFP and/or TdTomato in neural stem cells in the adult brain, mice ( $8-10$ weeks old) were given freshly made tamoxifen (dissolved in corn oil with $2 \%$ glacial acetic acid, $200 \mathrm{mg} / \mathrm{kg}$; Sigma-Aldrich) by oral gavage as described previously (Pan et al., 2012b,c). Briefly, for cellular studies, tamoxifen was administered once a day for $7 \mathrm{~d}$, whereas for behavior studies, it was given once a day for $4 \mathrm{~d}$ in each cycle, for three cycles with 2 week intercycle intervals. Bromodeoxyuridine (BrdU; Sigma-Aldrich) was dissolved in sterile saline with $0.007 \% \mathrm{NaOH}$ and administered at $100 \mathrm{mg} / \mathrm{kg}$ by intraperitoneal injection five times (every $2 \mathrm{~h}$ ) in $1 \mathrm{~d}$. Mice were killed either $2 \mathrm{~h}$ or 4 weeks after the last BrdU injection to identify BrdU-labeled proliferative cells or BrdU-retaining, adult-born cells.

Adult neural progenitor cell culture and in vitro analysis. Primary cell cultures were prepared using a neural tissue dissociation kit (Miltenyi Biotec) as previously described (Pan et al., 2013a). Briefly, tissue samples from caMEK5 knock-in mice containing the SVZ were microdissected and enzymatically digested with enzyme mixes provided by the dissociation kit. Tissue samples were mechanically triturated and DMEM/F-12 medium with $10 \%$ FBS was added to stop digestion. Tissue samples were then filtered through a cell strainer $(40 \mu \mathrm{m})$, spun down, washed once, and resuspended with DMEM/F-12 medium with $10 \%$ FBS and Percoll solution, followed by spinning down. Cell pellets were further resuspended and washed with initial proliferation medium (IPM; neurobasal medium with B27, L-glutamine, penicillin-streptomycin, $20 \mathrm{ng} / \mathrm{ml} \mathrm{EGF}$ and $10 \mathrm{ng} / \mathrm{ml} \mathrm{bFGF}$ ) and finally plated into $35 \mathrm{~mm}$ Petri dishes to permit neurosphere formation. Cells were maintained at $37^{\circ} \mathrm{C}$ with $6.5 \% \mathrm{CO}_{2}$ and cultured for $\sim 7 \mathrm{~d}$ until visible neurospheres formed. Growth factors (EGF and bFGF) were refreshed every 3-4 d. Spheres collected from secondary passage were dissociated and plated as a monolayer culture on fibronectin and poly-L-ornithine (BD Biosciences)-coated culture plates or Aclar coverslips (Electron Microscopy Sciences) for experiments.

For in vitro induction of caMEK5-eGFP expression, progenitor cells were infected with AAV viruses (control or Cre virus). Briefly, $1 \mathrm{~d}$ after plating, SVZ-aNPC cells were infected with AAV viruses $\left(1 \times 10^{10}\right.$ $\mathrm{vg} / \mathrm{ml}$ ). Forty-eight hours after infection, cells were then subjected to differentiation assays. IPM was replaced with medium free of EGF and bFGF for $5 \mathrm{~d}$, followed by fixation with $4 \%$ paraformaldehyde (PFA)/sucrose for $30 \mathrm{~min}$ at room temperature and immunocytochemical analysis.
Western blots. Adult neural progenitor cells were plated at a density of $1 \times 10^{6}$ cells per well in fibronectin and poly-L-ornithine-coated 12-well plates. At $24 \mathrm{~h}$ after plating, cells were infected with AAV viruses as described above. Cells were harvested $72 \mathrm{~h}$ after infection and then washed with cold PBS, pH 7.4, and lysed with Triton X lysis buffer. Cell lysates were clarified by centrifugation and protein concentration was determined using a BCA protein assay (Pierce). Samples containing 10 $\mu \mathrm{g}$ protein were separated on $10 \%$ SDS-PAGE gels and transferred to PVDF membrane (Millipore), followed by ECL Plus (GE Healthcare) detection. The following antibodies were used as primary antibodies for Western analysis: rabbit anti-ERK5 (1:20,000; Cavanaugh et al., 2001), rabbit anti-phospho-ERK5 (1:1000, Thr218/Tyr220; Cell Signaling Technology), rabbit anti-GFP (1:1000; Invitrogen), rabbit anti-MEK5 (1:1000; Enzo Life Sciences), and mouse anti- $\beta$-actin (1:20,000; Sigma).

Immunohistochemistry. Mice were killed and perfused transcardially with $4 \%$ PFA in PBS. Brains were postfixed in 4\% PFA overnight, and followed by $30 \%(\mathrm{w} / \mathrm{v})$ sucrose/PBS solution at $4^{\circ} \mathrm{C}$ until brains sank. Immunohistochemistry was processed as previously described (Wang et al., 2014). The following primary antibodies and dilutions were used for immunohistochemistry: rat monoclonal anti-BrdU (1:500, AbD; Serotec), mouse monoclonal anti-NeuN (1:500; Millipore), mouse monoclonal anti-Sox2 (1:500; Promega), mouse monoclonal anti-GFAP (1:1000; Millipore), goat polyclonal anti-OMP antibody (1:1000; Wako), rabbit polyclonal anti-Ki67 (1:200; Leica), rabbit polyclonal anti-Cleaved caspase-3 (1:200; Cell Signaling Technology), mouse monoclonal antiCalretinin (1:500; Santa Cruz Biotechnology), and mouse monoclonal anti-Reelin (1:2000; Abcam).

Confocal imaging and analysis. All images were captured with an Olympus Fluoview-1000 laser scanning confocal microscope with numerical aperture (NA) $0.75,10 \times, 20 \times$ lenses, or an NA $1.3,40 \times$ oilimmersion lens. Optical Z-sections $(0.5-1 \mu \mathrm{m})$ were collected and processed using ImageJ software (NIH). Images were uniformly adjusted for color, brightness, and contrast with Photoshop CS6 (Adobe Systems).

Quantification of immunostained cells. Every eighth serial coronal section $(30 \mu \mathrm{m})$ containing the SVZ or every fifth section containing the entire olfactory bulb $(30 \mu \mathrm{m})$ was immunostained. Immunoreactivepositive cells $\left(\mathrm{BrdU}^{+}, \mathrm{NeuN}^{+}\right.$, or double-BrdU $\left.{ }^{+} / \mathrm{NeuN}^{+}\right)$were counted with the experimenter blinded to mice genotypes or treatments as previously described (Pan et al., 2012a; Zou et al., 2012). For the SVZ, cells were counted manually and the resulting cell numbers were scaled relative to the number of sections per SVZ (Pan et al., 2012a). For the OB, stereology software (MBF Bioscience) was used as previously described to count cells (Zou et al., 2012). ImageJ was used for measuring Sox 2 and TdTomato immunofluorescence intensity. For tracing and measurement of dendritic length and branching numbers, Simple Neurite Tracer (NIH) was used as previously described (Li et al., 2013b). For colocalization analysis of BrdU and NeuN, a minimum of 200 immunopositive cells from each mouse were randomly selected, and the coexpression of $\mathrm{NeuN}$ and BrdU was determined by the presence of overlapping fluorescent signals in a Z-series stack.

Olfactory habituation/dishabituation test. This was conducted as previously described (Pan et al., 2012a; Zou et al., 2012). Briefly, animals were pretrained with mineral oil-laced cotton swabs for four $60 \mathrm{~s}$ presentations with $2 \mathrm{~min}$ intervals to ensure that subsequent exposure to an odorantlaced cotton swab did not elicit a response due to object novelty. The odor habituation/dishabituation tests were then conducted by presenting isoamyl acetate (IAA), citralva, and vanillin sequentially, with four presentations (60 s/presentation with $2 \mathrm{~min}$ intertrial interval) for each odorant. As we described previously, the duration of the animals' investigation (sniffing) of the cotton swab was recorded with a stopwatch (Pan et al., 2012a; Zou et al., 2012). The sniffing was defined as animals' noses approaching to and within $1 \mathrm{~cm}$ distance to the swabs (Pan et al., 2012a; Zou et al., 2012). A significant decrease in investigation time during subsequent presentations of the same odorant indicates odor recognition and habituation, and an increase in investigation of a new odorant indicates dishabituation (Pan et al., 2012a; Zou et al., 2012).

Threshold for odorant detection. This assay was performed as previously described (Pan et al., 2012a). Briefly, mice were presented with two cotton swabs simultaneously; one laced with mineral oil (vehicle control) 
and another with a specific odorant. The relative location of the two cotton swabs was randomly switched between presentations to avoid spatial learning. Mice were presented with ascending concentrations of the odorant on consecutive days with one concentration for each day. Each session lasted 3 min during which the total investigation time on each cotton swab was recorded. Data are presented as percentage of the time spent investigating the odorant over the total sniffing of both cotton swabs. Sniffing durations $>50 \%$ of the time indicates that mice detected the odorant (Pan et al., 2012a).

Olfactory short-term memory. This was performed as previously described (Pan et al., 2012a). Mice were presented with a cotton swab laced with the same odorant during two sessions ( $5 \mathrm{~min} /$ session) separated by $30,180,360$, and 480 min intervals with a different odorant used for each interval time point. Only one time interval was tested on each day to avoid cross interference of olfactory detection and memory. A significant decrease in total investigation time of the cotton swab during the second presentation of the odorant suggests olfactory memory for the first presentation of the same odorant (Pan et al., 2012a).

Sand-digging based odor-cued associative olfactory learning. Mice were food restricted to maintain $85-90 \%$ of free-feeding body weight for 4-5 $\mathrm{d}$ before the beginning and throughout the entire test (Pan et al., 2012a; Zou et al., 2012). The test was performed as previously described using the same apparatus (Zou et al., 2012). Before the test, mice were pretrained to learn to retrieve the food reward buried in sand. The pretraining session consisted of 3 consecutive days during which the food reward was put on top of the sand on day 1 , partially buried on day 2 , and deeply buried on day 3 with four trials in the morning block and four trials in the afternoon block ( 1 min intertrial interval and $4 \mathrm{~h}$ interblock interval) each day. By the end of pretraining on day 3 , mice would have learned to retrieve food reward by digging sand within $30 \mathrm{~s}$. The day after pretraining, mice were subjected to the olfactory discrimination test for four trials per block, two blocks per day for $8 \mathrm{~d}$. During the test, both dishes were filled with sand with one scented with limonene $(-)$ and another with limonene $(+)$. A food reward was buried at the bottom of the dish scented with limonene $(+)$. To avoid spatial cues, the two dishes were placed on either the left or the right side randomly as long as each dish was placed on each side twice per block but no more than three consecutive times each day. Scoring for correct or incorrect choice was based on the animal's first dig. If the mouse's first dig was correct, the mouse was allowed to finish until it retrieved the food reward. If the mouse's first dig was incorrect, it was allowed to finish that side but not to self-correct.

We also performed a "challenging" version of the sand-digging task with a different cohort where only one block (4 trials) of training was conducted each day for $10 \mathrm{~d}$. Instead of using one pair of structurally similar odorants, two pairs were used in the challenging assay: carvone $(+)$ versus carvone $(-)$ and butanol versus pentanol. The presentations of the two pairs were randomized each day with two presentations for each pair. Scoring for correct or incorrect choice was the same as the standard test.

Intranasal zinc sulfate delivery. Tamoxifen or vehicle was administered once per day for $4 \mathrm{~d}$ in each cycle for three cycles, with a 2 week intercycle interval, starting when mice were 3 months old. Mice were treated with zinc sulfate when they were $\sim 12$ months old. Zinc sulfate solution was prepared at $0.17 \mathrm{~m}$ using sterile saline $\left(\mathrm{ZnSO}_{4} \cdot 7 \mathrm{H}_{2} \mathrm{O}\right.$; Sigma-Aldrich). Adult male mice were anesthetized by intraperitoneal injection of ketamine and xylazine mixture. Anesthetized mice were laid face up and secured onto a Styrofoam board placed at a $45^{\circ}$ angle with the horizontal plane, and the head was orientated downward. A blunt-tipped HPLC syringe (Hamilton) was loaded with $\mathrm{ZnSO}_{4}$ solution and attached to a syringe pump (KDS-100). The syringe tip was directed to approach the nose and stopped when it just touched one nostril. Zinc solution (4-6 $\mu \mathrm{l})$ was slowly infused by the pump at a rate of $150 \mu \mathrm{l} / \mathrm{h}$. After the delivery to one nostril, mice were allowed to rest for $15 \mathrm{~min}$ before infusion of the other nostril. After receiving bilateral delivery, mice were kept on a warm pad until they recovered from anesthesia.

MOE tissue processing. After perfusion, main olfactory epithelium (MOE) was dissected following a previous method with modifications (Dunston et al., 2013). Briefly, the mandible and incisors were first cutoff. Molars and hard palate were removed, followed by zygomatic bones.
The bones covering the brain were removed as much as possible leaving only part of the frontal bones covering the OB. The partially deboned MOE and attached brain were postfixed in $4 \%$ PFA at $4^{\circ} \mathrm{C}$ overnight and decalcified in $0.5 \mathrm{M}$ EDTA, $\mathrm{pH} 8.0$, at $4^{\circ} \mathrm{C}$ for $\sim 1$ week. After decalcification, tissues were infiltrated by $30 \%$ sucrose in PBS and embedded in optimum cutting temperature (OCT) compound, and stored in $-80^{\circ} \mathrm{C}$.

Nissl staining. MOE sections were washed in PBS for $3 \times 5$ min to remove residual OCT followed by a brief wash in $\mathrm{dd}_{2} \mathrm{O}$. Slides were incubated in prewarmed $\left(50^{\circ} \mathrm{C}\right) 0.1 \%$ cresyl violet aqueous solution (Sigma-Aldrich) for $20 \mathrm{~min}$ at room temperature. After staining, slides were quickly dehydrated through an ascending gradient of ethanol, cleared by two changes of xylene, and coverslipped with Permount (Fisher Scientific).

Statistical analysis. All data were expressed as mean \pm SEM. Student's $t$ test (two-tailed) was used to analyze in vivo cellular data. For behavioral tests, ANOVA with repeated measures was used for the sand diggingbased test (see Fig. 13), and Student's $t$ test was used to analyze all other behavioral data. N.S., not significant; ${ }^{*} p<0.05,{ }^{* *} p<0.01$, ${ }^{* * *} p<0.001$.

\section{Results \\ caMEK5-eGFP is conditionally induced in the SVZ and OB regions of adult brain upon tamoxifen administration}

We previously generated an ERK5-activating knock-in mouse line in which a constitutive active MEK5 (caMEK5) sequence tagged with eGFP was inserted into the Gt(ROSA)26Sor genomic locus (ROSA26) through homologous recombination (Wang et al., 2014). To induce expression of caMEK5-eGFP in adult brains, the caMEK5-eGFP mice were bred with Nestin-CreER ${ }^{\mathrm{TM}}$ mice, an inducible Cre line driven by the Nestin promoter (Kuo et al., 2006). Mice obtained by treating Nestin-CreER ${ }^{\mathrm{TM}}$ :caMEK5eGFP with tamoxifen are designated as NesCreER:caMEK5 mice (Fig. 1A). Upon tamoxifen induction, Cre-mediated recombination and expression of caMEK5-eGFP in these mice occurs specifically in Nestin-expressing neural stem cells and their progeny. Expression levels of caMEK5-eGFP were similar to endogenous MEK5 (Wang et al., 2014). A 7 d tamoxifen treatment induced caMEK5-eGFP expression specifically in the SVZ and the OB regions (Fig. $1 D, E, H, I$ ), as well as in the subgranular zone of the dentate gyrus (DG; Wang et al., 2014). Vehicle-treated control mice did not show any GFP staining in these regions (Fig. $1 B, C, F, G)$.

We also examined the expression of caMEK5-eGFP in other regions of the adult brain. We found no expression in the cortex (Fig. $1 J, K$ ), striatum (Fig. $1 L, M$ ), cornu ammonis (CA) 1 (Fig. $1 N, O$ ), and CA3 of the hippocampus (Fig. $1 P, Q$ ), thalamus (Fig. $1 R, S$ ), or hypothalamus (Fig. 1T,U). Together with our recent report (Wang et al., 2014), these data demonstrate that caMEK5eGFP is expressed only in the adult neurogenic regions and only upon tamoxifen administration.

\section{The caMEK5-eGFP gene specifically activates ERK5 and increases adult neurogenesis in vitro}

To examine the function of caMEK5-eGFP fusion protein in adult neural progenitor cells (aNPCs), SVZ-derived aNPCs (SVZ-aNPCs) were prepared from 8-week-old caMEK5-eGFP ${ }^{\text {loxP/loxP }}$ mice and infected with AAV-Cre or its control virus (AAV-ctrl). The caMEK5-eGFP fusion protein was induced in cells infected with the AAV-Cre virus (Fig. 2A). The expression level of caMEK5-eGFP protein was comparable to that of the endogenous MEK5, suggesting that the transgene was not over expressed. Significantly, caMEK 5 expression activated endogenous ERK 5 as evidenced by ERK5 phosphorylation and a band shift in total ERK5 protein. However, it did not activate the structurally related ERK1/2, 
A

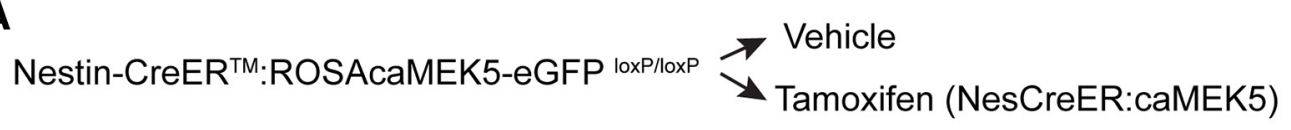

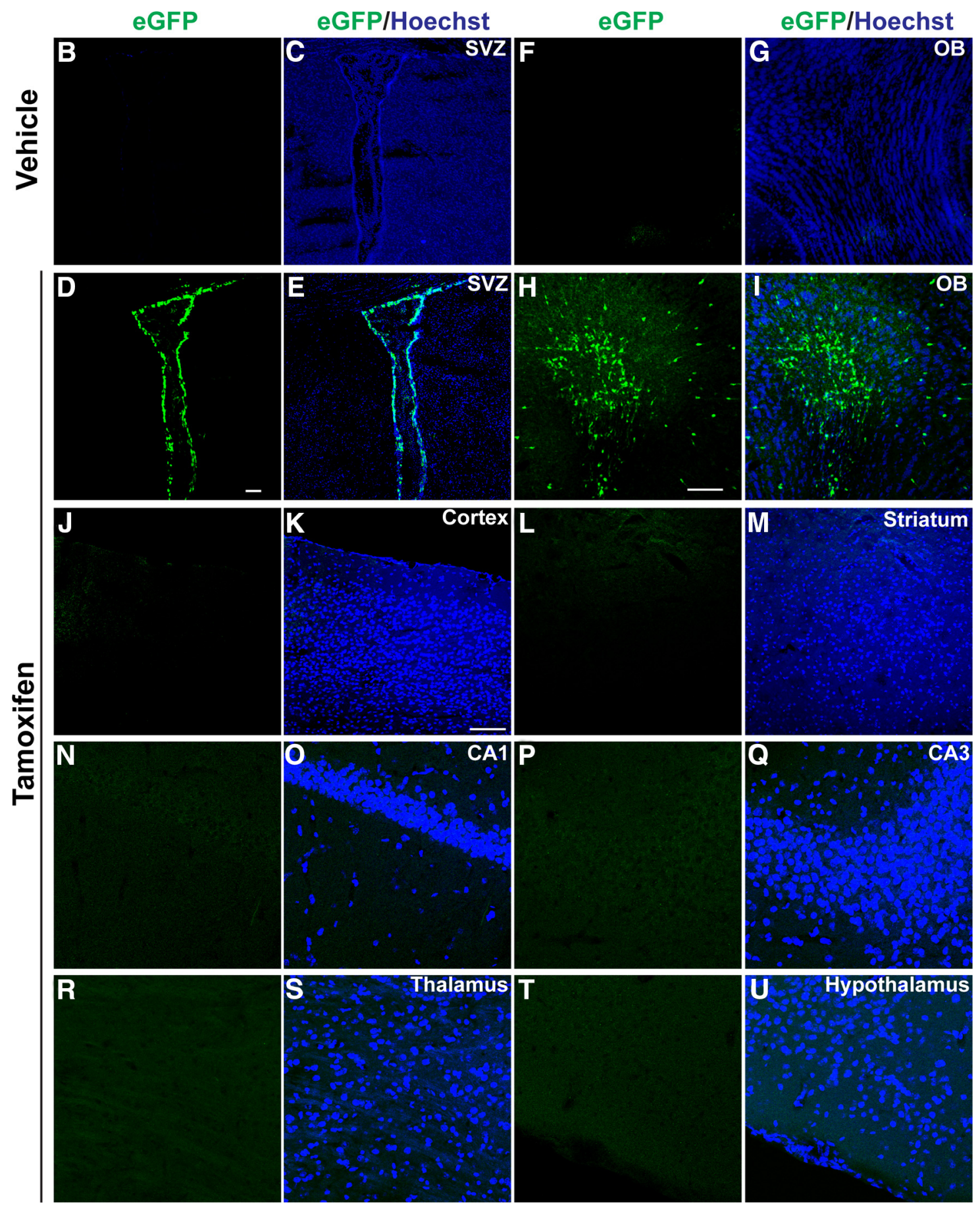

Figure 1. caMEK5-eGFP is conditionally induced in the SVZ and OB regions of adult brain in NesCreER:caMEK5 mice upon tamoxifen administration. $\boldsymbol{A}$, Experimental scheme for generating NesCreER:caMEK5 mice. Nestin-CreER ${ }^{{ }^{M}}$ :caMEK5-eGFP ${ }^{\text {loxP/loxP }}$ mice were generated by crossing (ROSA26) caMEK5-eGFP ${ }^{\text {loxP/loxP }}$ with Nestin-CreER ${ }^{{ }^{T M}}$ mice. Eight-week-old mice were treated with vehicle (corn oil) or tamoxifen by oral gavaging once a day for $7 \mathrm{~d}$ and perfused 3 weeks after the last tamoxifen dosing. $\boldsymbol{B}-\boldsymbol{I}$, Anti-eGFP immunostaining (green) shows the expression of caMEK5-eGFP in the SVZ of the lateral ventricle and the olfactory bulb of NesCreER:caMEK5 $(\boldsymbol{D}, \boldsymbol{E}, \boldsymbol{H}, \boldsymbol{I})$ but not the vehicle-treated control mice $(\boldsymbol{B}, \boldsymbol{C}, \boldsymbol{F}, \boldsymbol{G})$. Scale bars: $\boldsymbol{D}, \boldsymbol{H}, 100 \mu \mathrm{m}$; for panels of $S V Z$ and OB images, respectively. J-U, eGFP is not expressed in other regions of the adult brain including the cortex $(\boldsymbol{J}, \boldsymbol{K})$, striatum $(\boldsymbol{L}, \boldsymbol{M}), \mathrm{CA}(\boldsymbol{N}, \mathbf{O}), \mathrm{CA3}(\boldsymbol{P}, \boldsymbol{Q})$, thalamus $(\boldsymbol{R}, \boldsymbol{S})$, and hypothalamus $(\boldsymbol{T}$, U). Scale bars: J-U, $50 \mu \mathrm{m}$. 
A

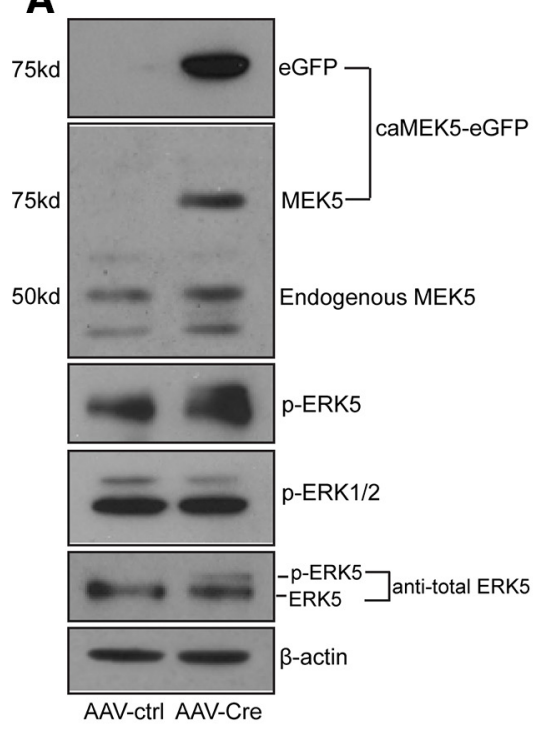

D

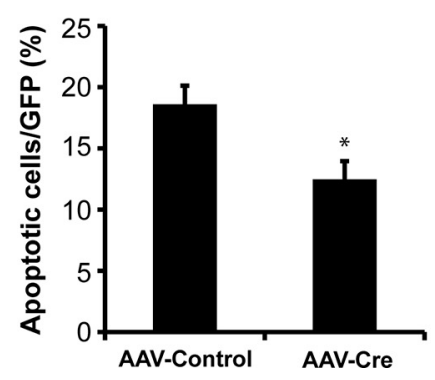

Figure 2. ERK5 activation increases adult neurogenesis in vitro. $\boldsymbol{A}$, Expression of caMEK5-eGFP fusion protein induced phos phorylation of endogenous ERK5 but not the closely related ERK1/2. aNPCs were cultured from the SVZ of caMEK5 ${ }^{\text {lox/lox }}$ mouse brain and infected with AAV-Cre or control (AAV-ctrl) viruses. Western blot analysis demonstrates expression of caMEK5-eGFP fusion protein in SVZ-aNPCs infected with AAV-Cre virus but not that infected with AAV-control virus. The anti-MEK5 antibody recognizes both endogenous MEK5 and caMEK5-eGFP fusion proteins. Phosphorylated ERK5 ( $p$-ERK5) and ERK1/2 (p-ERK1/2) were detected by an antibody that recognizes both on the same blot. Total ERK5 and $\beta$-actin were used as loading controls. The band shift in total ERK5 also indicates ERK5 activation. $\boldsymbol{B}$, ERK5 activation promotes neuronal differentiation in cultured SVZ-aNPCS. Viral infected SVZ-aNPCs were cultured under differentiation conditions for $5 \mathrm{~d}$. Cells were then fixed and immunostained for GFP to identify viral infected cells and for $\beta$-III tubulin, a marker for immature neurons. Scale bar, $25 \mu \mathrm{m}$. C, Quantification of neuronal differentiation ( $\beta$-III tubulin expressing cells among viral infected cells). $\boldsymbol{D}$, Quantification of apoptosis identified by nuclear fragmentation and/or condensation, among virus-infected cells. Virus-infected SVZ-aNPCs were cultured under differentiation conditions for $5 \mathrm{~d}$; ${ }^{*} p<0.05,{ }^{* *} p<0.01$.

demonstrating the specificity of caMEK5. We then examined whether ERK5 activation promotes SVZ-aNPCs neurogenesis. Two days after AAV virus infection, SVZ-aNPC cells were maintained under differentiation conditions for $5 \mathrm{~d}$. Cultures infected with AAV-Cre virus contained a significantly higher percentage of cells expressing $\beta$-III tubulin ( $p=0.0089$; Fig. $2 B, C$ ), a marker for newborn neurons, compared with the cultures infected with the control virus. Furthermore, there were fewer apoptotic cells when the culture was infected with AAV-Cre than that with AAVcontrol virus ( $p=0.024$; Fig. $2 D)$. These data suggest that ERK5 activation promotes neuronal differentiation and enhances cell survival of SVZ-aNPCs.

Inducible and conditional activation of ERK5 increases adult olfactory neurogenesis in vivo

We examined the effect of caMEK5 on adult olfactory neurogenesis in vivo using $\mathrm{OB}$ sections from NesCreER:caMEK5 mice, littermate tamoxifen control (caMEK5-eGFP ${ }^{\text {loxP/loxP }}$ mice treated with tamoxifen, designated as "control" hereafter), and littermate vehicle control (Nestin-CreER ${ }^{\mathrm{TM}}$ :caMEK5-eGFP mice treated with corn oil, designated as "vehicle" hereafter; Fig. $3 A$ ). Mice were treated daily with vehicle or tamoxifen for $7 \mathrm{~d}$. To label adult-born cells, BrdU was injected five times (every $2 \mathrm{~h}$ for $10 \mathrm{~h}$ ) on the fourth day after the last dose of tamoxifen, and mice were perfused 4 weeks after BrdU administration. The total number of $\mathrm{BrdU}^{+}$cells in the granular cell layer (GCL) of the OB of NesCreER:caMEK5 mice was significantly higher than that of control $(p=0.038)$ and vehicle $(p=$ 0.045; Fig. $3 B-G, N)$. Furthermore, an increased number of adult-born, mature neurons $\left(\mathrm{NeuN}^{+}\right.$and $\mathrm{BrdU}^{+}$colabeled cells) was observed in the GCL of the OB of NesCreER:caMEK5 mice (Fig. $4 H_{-}$ $M, O ; p=0.013$ compared with control and $p=0.026$ compared with vehicle). We also compared the ratio of $\mathrm{BrdU}^{+}$, $\mathrm{NeuN}^{+}$cells over total BrdU ${ }^{+}$cells and found a significant increase in NesCreER: caMEK5 mice (Fig. $3 P$; $p=0.025$ compared with control and $p=0.010$ compared with vehicle), indicating an increase in neuronal differentiation among adult born cells.

We sought to investigate whether caMEK5 activation of ERK5 stimulates cell proliferation in the SVZ. Control and NesCreER:caMEK5 mice were injected with BrdU every $2 \mathrm{~h}$ for a total of five times and perfused $2 \mathrm{~h}$ after the last injection. There was no significant increase of $\mathrm{BrdU}^{+}$cells in the SVZ of NesCreER: caMEK5 mice compared with control littermates ( $p=0.30$; Fig. $4 A-E)$, suggesting that increased proliferation is unlikely the mechanism for caMEK5induced adult neurogenesis. Consequently, the increase of total adult born cells in the $\mathrm{OB}$ of NesCreER:caMEK5 mice is likely due to enhanced cell survival.

We previously found that ERK5 activation did not affect the adult neural stem cell pool in the DG (Wang et al., 2014). Nevertheless, we evaluated whether this is also the case for the SVZ. Analysis of SOX2, a marker for stem cells, showed no significant difference between NesCreER:caMEK5 and control mice that were perfused 4 weeks after tamoxifen treatment ( $p=0.76$; Fig. $4 F-J)$. Analysis of another stem cell marker, GFAP, also indicates similar results (data not shown). These results indicate that activation of endogenous ERK5 by caMEK5 does not affect the adult neural stem cell pool along the SVZ. Together, these data suggest that activation of ERK5 along the SVZ increases adult olfactory neurogenesis by promoting cell survival and neuronal differentiation.

ERK5 activation promotes neuronal maturation in the $\mathrm{OB}$ We examined whether activation of ERK5 promotes neuronal maturation. To facilitate this study, male Nestin-CreER ${ }^{\text {TM }}$ :(ROSA) 
A $\left.\begin{array}{rl}\text { ROSA caMEK5-eGFP loxPlloxP } & \stackrel{\text { Tamoxifen }}{\longrightarrow} \text { Control } \\ \text { Nestin-CreER } & \stackrel{\text { Vehicle }}{\longrightarrow} \text { ROSA caMEK5-eGFP loxPlloxP } \\ \stackrel{\text { Tamoxifen }}{\longrightarrow} \text { Vehicle }\end{array}\right]$ NesCreER:cantrols
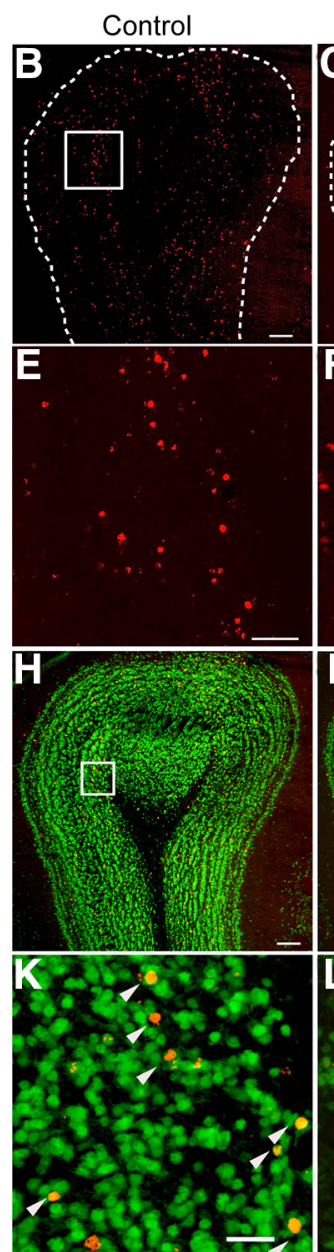

$\mathrm{BrdU} / \mathrm{NeuN}$

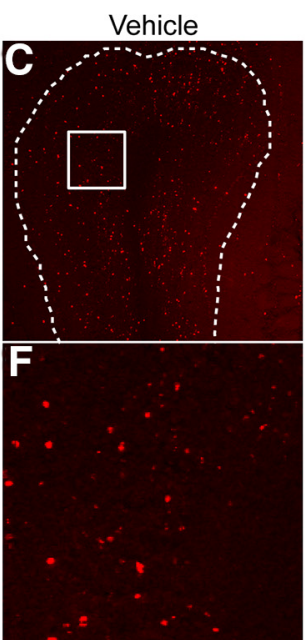

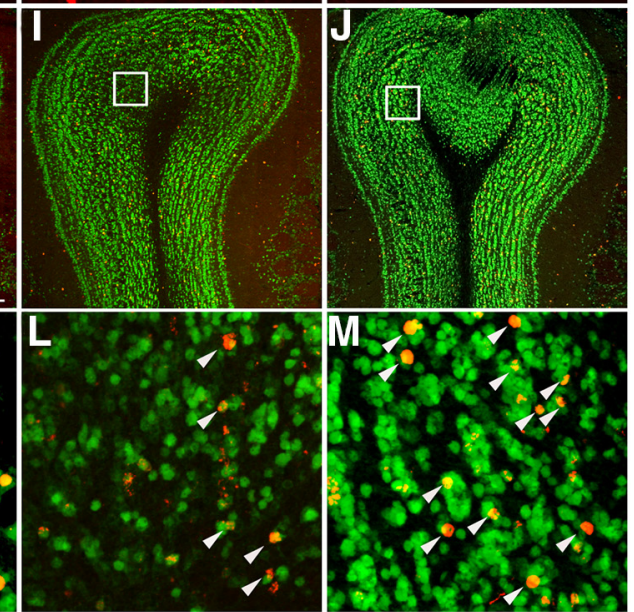
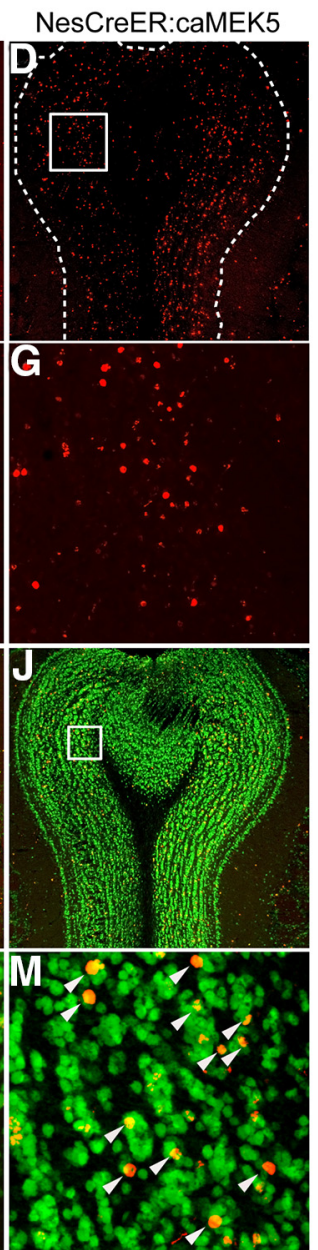

$\mathbf{N}$
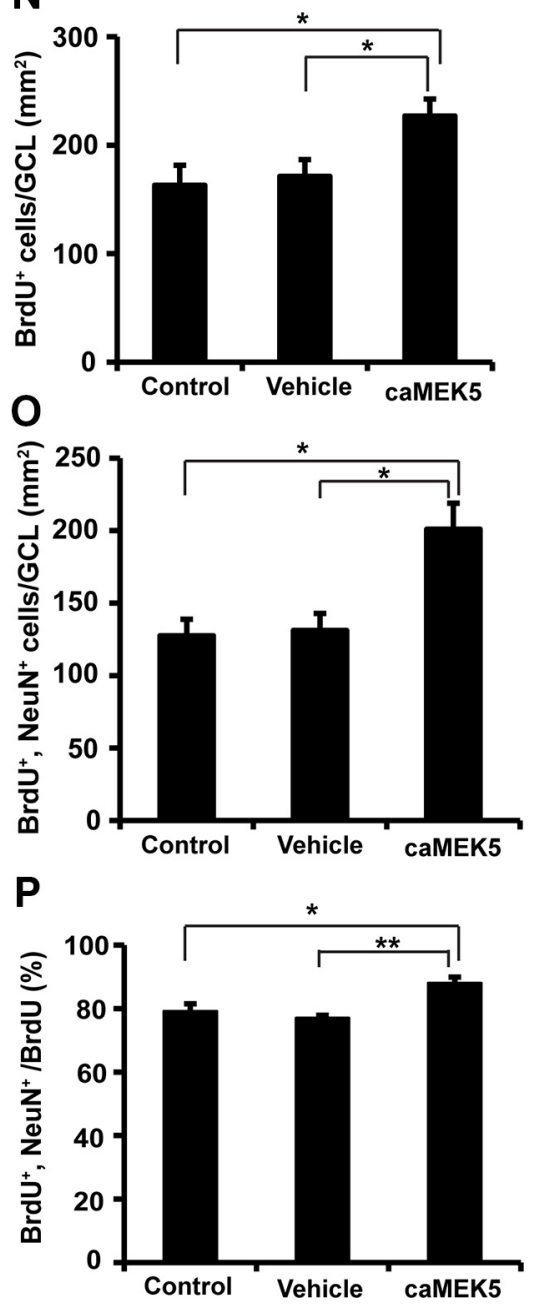

Figure 3. ERK5 conditional activation in the adult neurogenic regions enhances olfactory neurogenesis in the GCL of the OB in vivo. $A$, Experimental scheme for generating NesCreER:caMEK5 and control littermates (tamoxifen control and vehicle control). $\boldsymbol{B}-\boldsymbol{M}$, Representative BrdU (red) and NeuN (green) immunostaining images from the $0 B$ of (tamoxifen), control ( $\boldsymbol{B}, \boldsymbol{E}, \boldsymbol{H}, \boldsymbol{K})$, vehicle control $(\boldsymbol{C}, \boldsymbol{F}, \boldsymbol{I}, \boldsymbol{L})$, and NesCreER:caMEK5 $(\boldsymbol{D}, \boldsymbol{G}, \boldsymbol{J}, \boldsymbol{M})$ mouse brains. Mice were perfused 4 weeks after BrdU injection. Images in $\boldsymbol{E}-\boldsymbol{G}$ are enlarged areas corresponding to boxed regions in $\boldsymbol{B}-\boldsymbol{D}$, whereas images in $\boldsymbol{K}-\boldsymbol{M}$ are enlarged from boxed regions in $\boldsymbol{H}-\boldsymbol{J}$, respectively. Scale bars: $\boldsymbol{B}-\boldsymbol{D}, \boldsymbol{H}-\boldsymbol{J}, 100 \mu \mathrm{m} ; \boldsymbol{E}-\boldsymbol{G}, 50 \mu \mathrm{m} ; \boldsymbol{K}-\boldsymbol{M}, 25 \mu \mathrm{m} . \boldsymbol{N}, \mathbf{Q}$ uantification of total BrdU + cells in the $\mathrm{GCL}$ of each

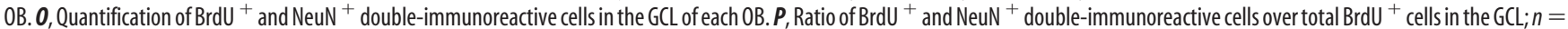
$3-4$ mice/group; ${ }^{*} p<0.05,{ }^{* *} p<0.01$.

caMEK5-eGFP ${ }^{\text {loxP/+ }}$ mice were crossed with a transgenic reporter line, (ROSA26) floxed-stop TdTomato, which expresses the bright fluorescent protein TdTomato (TdT) from the same genomic locus (Gt(ROSA)26Sor; Madisen et al., 2010) as the caMEK5-eGFP protein, upon Cre-mediated recombination. Mice treated with tamoxifen of the resulting line, Nestin-CreER ${ }^{\mathrm{TM}}$ :caMEK5-eGF$\mathrm{P}^{\text {loxP }}$ :TdTomato ${ }^{\text {loxP }}$ were designated "NesCreER:caMEK5/TdT", whereas similarly treated Nestin-CreER ${ }^{\mathrm{TM}}$ :TdTomato ${ }^{\text {loxP }}$ littermates, which lack caMEK5-eGFP loxP, were designated "NesCreER: TdT" and used as controls (Fig. 5A). Mice were treated with tamoxifen for $7 \mathrm{~d}$ and perfused 7 weeks after the last dose of tamoxifen.

We also characterized TdTomato $(+)$ cells with various cell specific markers. From IHC staining, we observed that TdTomato and caMEK5-eGFP are expressed in the same pattern both in the SVZ (Fig. 5B-E) and the OB (Fig. 5F-I). Furthermore, all caMEK5-eGFP $(+)$ cells coexpress TdTomato. TdTomato $(+)$ cells are more abundant and brighter than caMEK5-eGFP $(+)$ cells. Although we cannot rule out the possibility that TdTomato has a higher recombination efficiency than caMEK5-eGFP, the greater abundance of TdTomato $(+)$ cells is likely due to the fact that TdTomato is known as a superbly bright maker, whereas eGFP is expressed as a fusion tag with caMEK5, which depresses the immunohistochemical staining of the eGFP. Indeed, TdTomato is so bright that we can visualize its expression in tissue sections without antibody staining, whereas visualization of eGFP $(+)$ cells required an enhanced staining technique (tyramide signal amplification). Nevertheless, our Western blot data shown in Figure 2, as well as in our previous study (Wang et al., 2014), demonstrated a moderate expression 

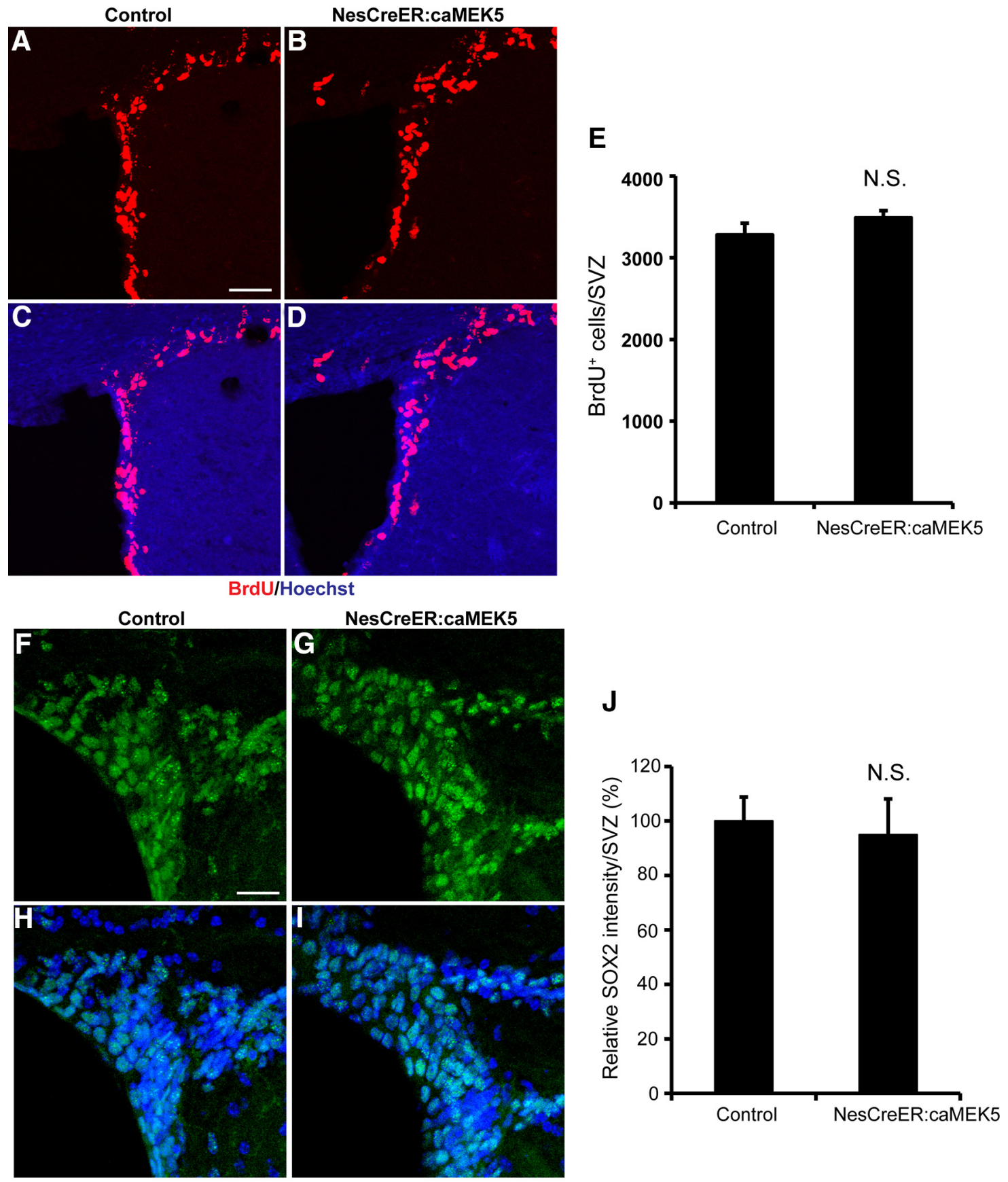

SOX2/Hoechst

Figure 4. Inducible and conditional caMEK5 activation of ERK5 does not affect cell proliferation or adult stem cell pool. $\boldsymbol{A}-\boldsymbol{D}$, Representative images of BrdU (red) staining in the SVZ of control ( $\boldsymbol{A}$, $\boldsymbol{C}$ ) and NesCreER:caMEK5 mice $(\boldsymbol{B}, \boldsymbol{D})$. Hoechst (blue) stains all nuclei. Scale bars, $50 \mu \mathrm{m}$. $\boldsymbol{E}$, Quantification of total BrdU ${ }^{+}$cells along the SVZ of control and NesCreER:caMEK5 mice. Control and NesCreER:caMEK5 mice were injected with BrdU every $2 \mathrm{~h}$ for a total of five times and perfused $2 \mathrm{~h}$ after the last injection; $n=3 \sim 4$ mice/group. $\boldsymbol{F}$ - , Representative images of $S$ ox2 (green) staining in the SVZ of control $(\boldsymbol{F}, \boldsymbol{H})$ and NesCreER:caMEK5 mice $(\boldsymbol{G}, \boldsymbol{I})$. Scale bar, $50 \mu \mathrm{m}$. J, Measurements of relative Sox2-immunoreactive signal intensity along the SVZ of NesCreER:caMEK5 and control mice that were perfused 4 weeks after tamoxifen treatment; $n=3$ mice/group. N.S., Not statistically significant.

of caMEK5-eGFP protein in the two adult neurogenic regions (the SGZ of the DG and the SVZ) and at similar abundance as the endogenous MEK5. Since all caMEK5-eGFP cells express TdTomato and TdTomato is technically more feasible for multiplemarker costaining, we used TdTomato as a surrogate reporter to characterize cells that have undergone Cre-mediated recombination in the SVZ, RMS, and OB. TdTomato $(+)$ should be a valid maker for recombination for comparing control mice with caMEK5-eGFP mice. If anything, using TdTomato as a reporter would only underestimate the effect of caMEK5.
We performed immunohistochemistry of the SVZ sections from NesCreER:TdT mice for coexpression of TdTomato and various cell markers. We found that TdTomato was rarely expressed in quiescent stem cells (GFAP+; Fig. 6A) or mature neurons (NeuN+; Fig. 6E). However, it was expressed in almost all proliferating stem/precursor cells (SOX2+; Fig. 6B), neuroblasts/immature neurons (DCX+; Fig. 6C), and proliferating cells (Ki67+; Fig. 6D). These data indicate that the NestinCreER $^{\mathrm{TM}}$-driven recombination takes place in stem/precursor cells in the SVZ. 
A

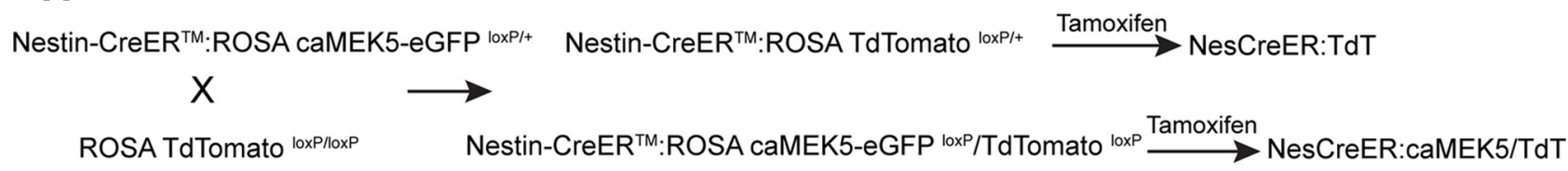

NesCreER:caMEK5/TdT
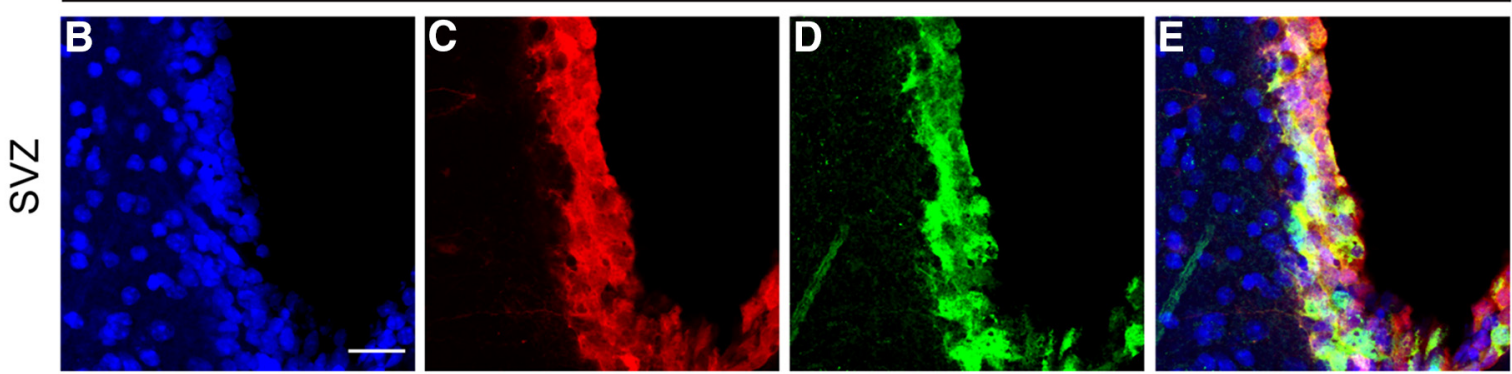

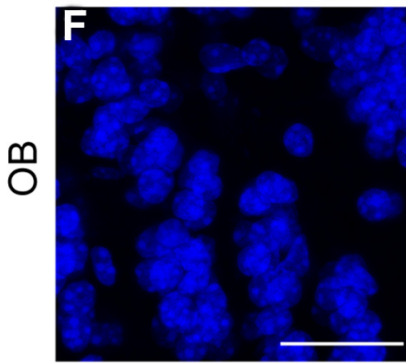

Hoechst

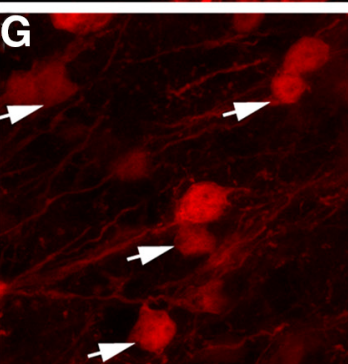

TdT

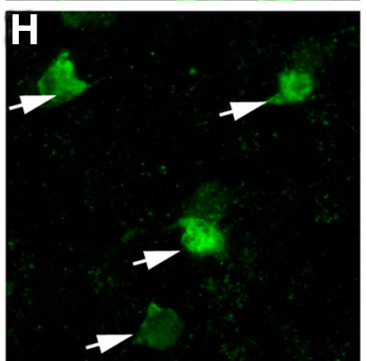

eGFP

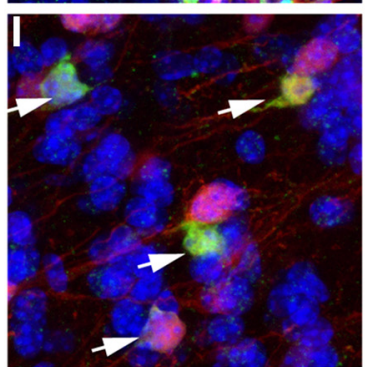

Merge

Figure 5. Crossing of a reporter line (ROSA26 TdTomato ${ }^{\text {loxP/loxP }}$ ) with NesCreER:caMEK5 to facilitate cellular characterization. $\boldsymbol{A}$, Breeding and tamoxifen treatment scheme for generating NesCreER:caMEK5/TdT mice and NesCreER:TdT control littermates. $\boldsymbol{B}-\boldsymbol{E}$, Representative confocal images of TdTomato (red) and eGFP (green) expression in the SVZ of NesCreER:caMEK5/TdT. Mice were treated with tamoxifen daily for $7 \mathrm{~d}$ and perfused 7 weeks later. $\boldsymbol{F}-\boldsymbol{I}$, Representative TdTomato (red) and eGFP (green) expression in the 0B of NesCreER:caMEK5/TdT. Arrows indicate cells coexpressing TdT and eGFP. Scale bars, $25 \mu \mathrm{m}$.

TdTomato was expressed in proliferating cells $(\mathrm{BrdU}+)$ in both the RMS (Fig. $6 F$ ) and OB (data not shown); almost $80 \%$ of $\mathrm{BrdU}+$ cells were TdTomato + , suggesting high recombination efficiency in newborn cells. TdTomato was also expressed in neuroblasts/immature neurons (DCX+; Fig. 6G), but not in mature neurons $(\mathrm{NeuN}+$ ) in the RMS (Fig. 6H). BrdU+ cells in the RMS and $\mathrm{OB}$ In the $\mathrm{OB}$, TdTomato was primarily expressed in interneurons (calretinin + and $\mathrm{NeuN}+$; Fig. $6 I, L$ ) and immature neurons (DCX+; Fig. $6 \mathrm{~K}$ ), but not in mitral cells (Reelin+; Fig. $6 J)$. Together, these data suggest that the Nestin-CreER ${ }^{\mathrm{TM}}$ driven recombination occurred in the stem/precursor cells (SVZ) and their progeny in the RMS, resulting in an increasing number of newly formed interneurons in the OB. These data are consistent with another study using a similar Nestin-Cre ${ }^{\text {ER }}$ line (Nestin-CreER ${ }^{\mathrm{T} 2}$; Lagace et al., 2007).

Because the expression of TdTomato facilitates the visualization of morphological changes of adult-born neurons in the $\mathrm{OB}$ of both groups, we further examined neuronal maturation in the $\mathrm{OB}$ using TdTomato as a reporter. The dendritic length and branching number were quantified with a simple neurite tracer as a measurement of neuronal maturation (Li et al., 2013b). Activation of ERK5 significantly increased the dendritic length $(p=$ 0.017 ; Fig. $7 A-E)$ and the branching of adult-born neurons ( $p=$ 0.032; Fig. $7 A-D, F)$.

Earlier data in Figure 4 implied that the increase of total adultborn cells in the OB of NesCreER:caMEK5 mice is likely due to enhanced cell survival. Consequently, we examined the ex- pression of active caspase-3, a marker for apoptosis, together with TdTomato reporter in the OB of NesCreER:caMEK5/ TdT and control NesCreER:TdT littermates. There were fewer caspase- $3+/ \mathrm{TdT}+$ cells in the OB of NesCreER:caMEK5/TdT mice than in the controls $(p=0.041$; Fig. 8$)$, confirming that the increase in the number of adult-born neurons in NesCreER:caMEK5 mice is at least partly due to enhanced cell survival in the OB.

Neuroblasts of the SVZ migrate tangentially along the RMS to the $\mathrm{OB}$ (chain migration), where they then switch orientation and migrate radially to the different layers of the OB. We examined whether ERK5 activation affects the chain migration of adult-born neurons in the RMS. Mice were treated with tamoxifen for $7 \mathrm{~d}$ and perfused $10 \mathrm{~d}$ after the last dose of tamoxifen. Chain migration was examined by TdTomato expression on sagittal sections, which showed that newborn cells in the RMS of NesCreER:caMEK5/TdT and control NesCreER:TdT mice have similar morphology, are elongated with leading processes and lined up orderly parallel to the RMS (Fig. 9A,B). Quantification of TdTomato fluorescence intensity along the RMS also did not reveal any significant difference $(p=0.73$; Fig. $9 C)$, suggesting that no cells were abnormally accumulated in the RMS. We also examined radial migration in the $\mathrm{OB}$ on coronal sections and did not find any significant difference between the two groups. Both groups showed well arrayed migration in the OB; newborn cells radially migrating away from the core of the olfactory bulb have 


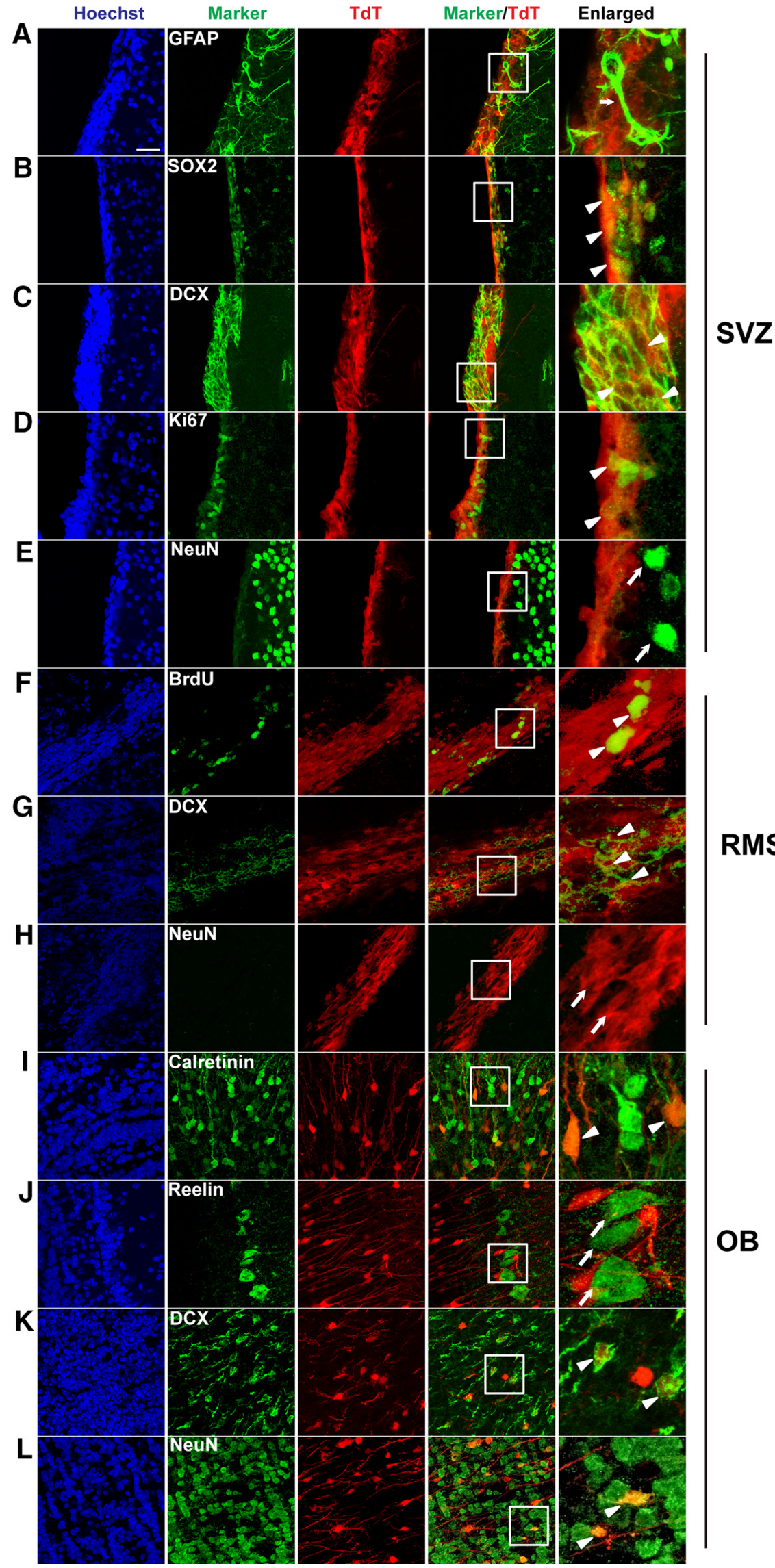

Figure 6. Characterization of Nestin-CreER ${ }^{\mathrm{TM}}$-mediated recombination in the SVZ, RMS, and OB. A-E, Representative confocal images of coronal SVZ sections stained with GFAP $(\boldsymbol{A})$, SOX2 (B), DCX (C), Ki67 (D), and NeuN (E) together with TdT (red) and similar morphology, migration orientation, and migration pattern (Fig. 9D).

NesCreER:caMEK5 mice show normal habituation/dishabituation olfactory behavior

The NesCreER:caMEK5 mice maintain normal general exploratory activity, motivation to explore, and do not show increased anxiety in the open-field assay (Wang et al., 2014). To investigate the effect of conditional caMEK5 stimulation of adult neurogenesis on olfactory behavior, several different cohorts of mice were used, including either the control mice, the vehicle mice as defined in Figure $3 A$, or both as controls. We first performed an odor habituation/dishabituation test, an assay to measure animals' ability to sense odorants. We used mineral oil (vehicle) and three distinct odorants: IAA, citralva, and ethyl vanillin in this assay. NesCreER: caMEK5 mice showed normal habituation/dishabituation behavior, which was similar to the control mice (Fig. 10). To examine the sensitivity of odor detection with an odor threshold assay, NesCreER: caMEK 5 mice and both groups of control mice were presented with a pair of cotton swabs laced with mineral oil or increasing concentrations of 1-octanol $(0.1-50 \mu \mathrm{M})$. NesCreER:caMEK5 mice performed similarly to the control and vehicle mice (Fig. 11). NesCreER:caMEK5 mice also showed similar sensitivity toward another odorant, ethyl acetate $(0.1 \mu \mathrm{M}$ to $10 \mathrm{~mm})$, as control mice (data not shown). Thus, caMEK5 stimulation of adult neurogenesis did not seem to affect the threshold for odorant detection.

\section{The NesCreER:caMEK5 mice show} improved short-term olfactory memory To examine the effect of caMEK5 on the formation of short-term olfactory memory, the NesCreER:caMEK5 and control mice were subjected to a cotton tip-based olfactory memory test. Mice were presented with the same odorant twice with an interval between the two presentations. The duration of sniffing at both presentations was scored and a significant decrease

Hoechst (blue). $\boldsymbol{F}-\boldsymbol{H}$, Representative images of sagittal RMS sections stained with $\operatorname{BrdU}(\boldsymbol{F}), \mathrm{DCX}(\boldsymbol{G})$, and $\operatorname{NeuN}(\boldsymbol{H})$ together with TdT (red) and Hoechst (blue). I-L, Representative images of coronal $O B$ sections stained with calretinin $(I)$, Reelin $(J)$, DCX $(\boldsymbol{K})$, and NeuN $(\boldsymbol{L})$ together with TdT (red) and Hoechst (blue). Right column represents the enlarged boxed areas respective to their left panels. Arrowheads point to colabeled cells whereas arrows represent TdT $(+)$ cells that do not express the marker. Scale bars, $25 \mu \mathrm{m}$. 

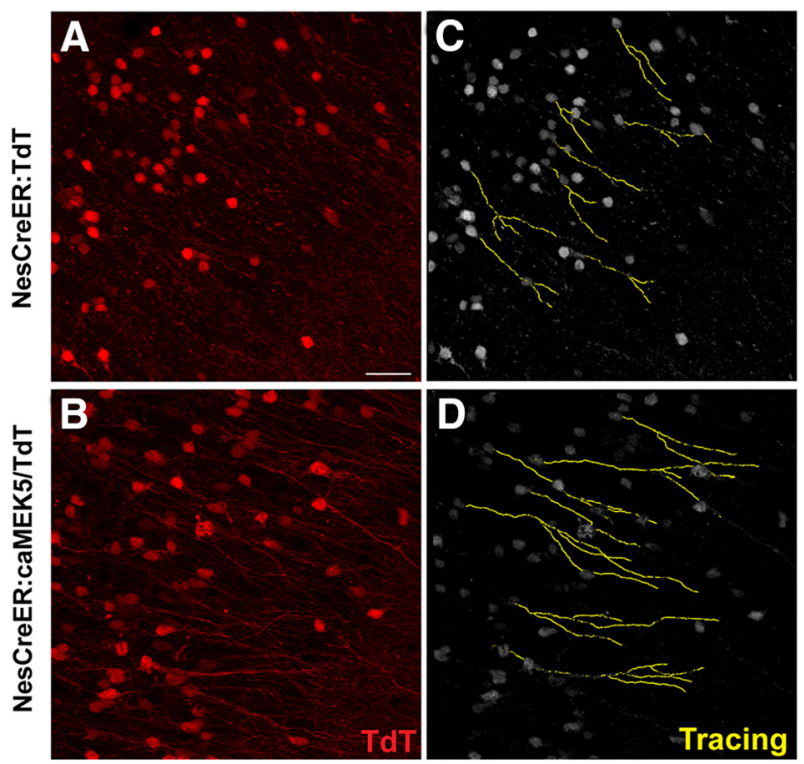
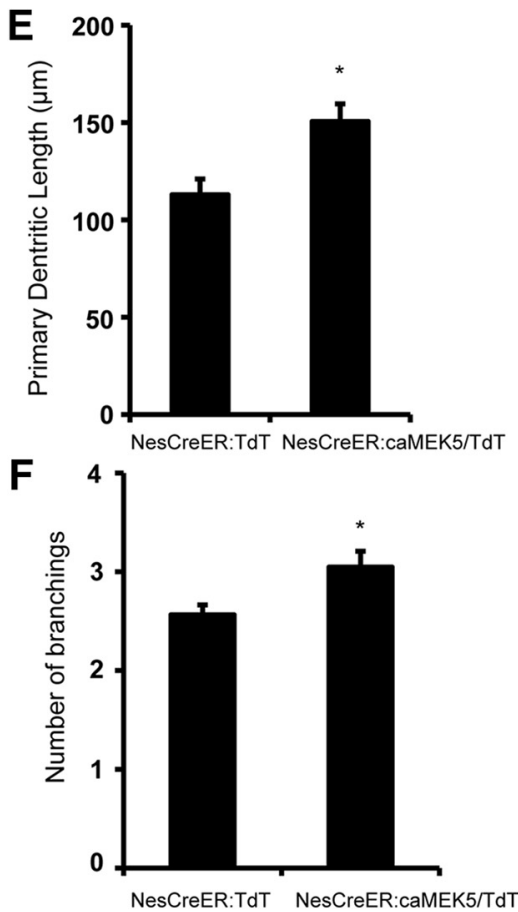

Figure 7. Inducible and conditional activation of ERK5 increases dendritic length and number of the branchings of adult-born neurons in the $0 B$. $A, B$, Representative TdTomato (red) expression from the OB of NesCreER:TdT $(\boldsymbol{A})$ and NesCreER:caMEK5/TdT $(\boldsymbol{B})$ mouse brains. Mice were treated with tamoxifen daily for $7 \mathrm{~d}$ and perfused 7 weeks later. $\boldsymbol{C}, \boldsymbol{D}, 3-\mathrm{D}$ tracing images of $\boldsymbol{A}$ and $\boldsymbol{B}$, respectively. Dendrites were highlighted by yellow color using Simple Neurite Tracer of ImageJ. Scale bar, $25 \mu \mathrm{m}$. $\boldsymbol{E}$, Average dendritic length of TdT ${ }^{+}$cells in the $0 B$ was measured. $\boldsymbol{F}$, Average number of dendritic branching of $\mathrm{TdT}^{+}$cells was measured; $n=4-5$ mice/group; ${ }^{*} p<0.05$.

in investigation during the second presentation compared with the first was used as a measurement of olfactory memory. Mice generally show less interest in odorants that they remember having encountered previously. Mice were tested at four intervals (30, 180, 360, and $480 \mathrm{~min})$, and different novel odorants were used for each time point. Both control and NesCreER:caMEK5 mice spent significantly less time sniffing the odor-laced cotton swab during the second exposure than the first exposure when the same odorant was presented $30 \mathrm{~min}$ apart $(p=0.0023$ for control and $p=0.010$ for NesCreER:caMEK5; Fig. $12 A, B)$. A similar trend was found for the $180 \mathrm{~min}$ interval $(p=0.012$ for control and $p=0.0048$ for NesCreER:caMEK5; Fig. 12 A, B), suggesting that both groups of mice maintained a short-term odorant memory for $30 \mathrm{~min}$ and $180 \mathrm{~min}$. However, the NesCreER: caMEK5 mice still exhibited significant olfactory memory at the $360 \mathrm{~min}(p=0.021)$ and $480 \mathrm{~min}(p=0.041)$ intervals, whereas the control mice did not ( $p=0.12$ for 360 min interval, $p=0.43$ for $480 \mathrm{~min}$ interval; Fig. 12A,B). These data suggest that NesCreER:caMEK5 mice have enhanced short-term olfactory memory.

\section{NesCreER:caMEK5 mice exhibit enhanced associative olfactory learning}

To determine whether caMEK5 stimulation of adult olfactory neurogenesis improves olfactory learning, we subjected mice to a sand digging-based, odor-cued associative olfactory learning assay. Before the test, mice were pretrained for $3 \mathrm{~d}$ with eight trials a day to associate food reward with sand digging. Both control mice and NesCreER:caMEK 5 mice learned the task by the end of pretraining (Fig. 13A). In the subsequent assay, to retrieve the food reward, the animals had to use olfactory cues to discriminate between a pair of structurally similar odorants: limonene $(+)$ versus limonene $(-)$ with only limonene $(+)$ associated with food reward. Although both control and NesCreER:caMEK5 mice learned the task by retrieving the food reward correctly in $>80 \%$ of the trials at the end of the training, NesCreER:caMEK5 performed significantly better than the control mice through the $8 \mathrm{~d}$ period (ANOVA with repeated measures; $F_{(1,15)}=14.42, p=$ 0.002; Fig. 13B).

We further tested the mice with an even more challenging form of odor-cued olfactory associated learning by training the animals 4 trials a day with 2 pairs of structurally similar odorants. We used a separate cohort of mice for this assay and also included the vehicle control group to address any concerns about the possible effect of Nestin-Cre transgene. At the end of a $3 \mathrm{~d}$ pretraining, both groups of control mice, as well as NesCreER:caMEK5 mice, learned the task of retrieving food from the dish by sand digging (Fig. 13C). Mice were then introduced with two pairs of odorants, carvone $(+)$ versus carvone $(-)$ and butanol versus pentanol. Each pair was presented for two trials a day, but the order of pair was randomized during the $10 \mathrm{~d}$ of training. The food reward was associated only with carvone $(+)$ or butanol. Over the course of $10 \mathrm{~d}$ of training, NesCreER: caMEK5 mice performed significantly better than either groups of control mice (control and vehicle) on days 9 and 10 (Fig. 13D), suggesting better odor-cued associative olfactory learning.

\section{Long-term olfactory memory is not altered in NesCreER:caMEK5 mice}

To determine whether enhanced adult neurogenesis impacts long-term olfactory memory, both the first and second cohorts of mice in Figure 13 were retested $30 \mathrm{~d}$ (Fig. 14A) or $21 \mathrm{~d}$ (Fig. 14B), respectively, after their last day of training for odor-cued associative olfactory learning. No significant difference in retrieving food reward was found between NesCreER:caMEK5 mice and 


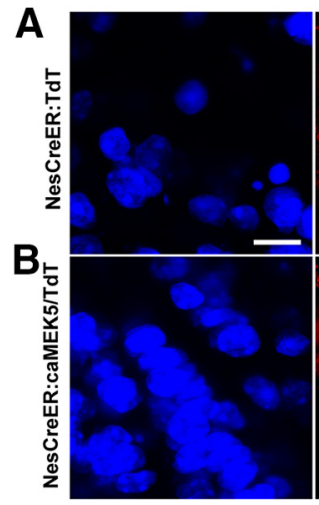

Hoechst

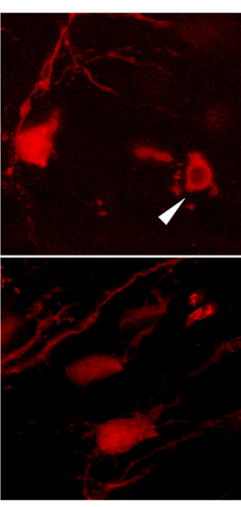

TdT

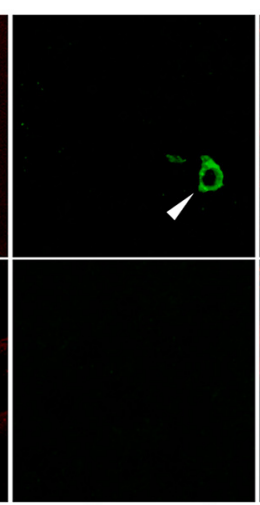

Caspase-3

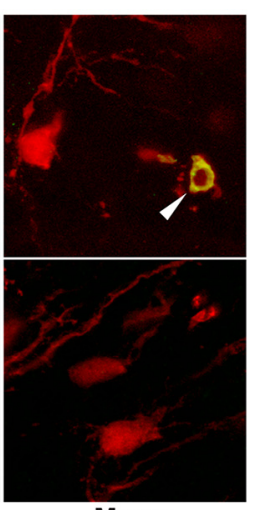

Merge

\section{C}

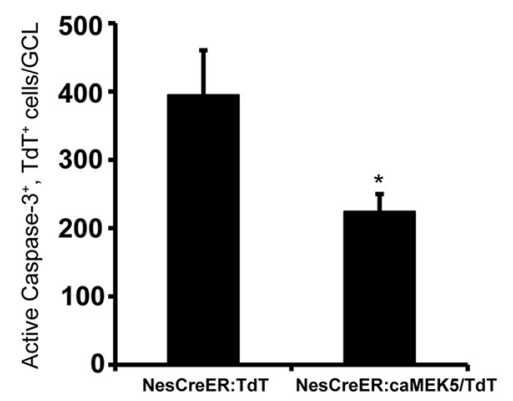

Figure 8. Inducible and conditional activation of ERK5 enhances cell survival in the OB. $A, B$, Representative confocal images of cells coexpressing TdT (red) and active caspase-3 (green) in the OB of NesCreER:TdT ( $\boldsymbol{A}$ ) and NesCreER:caMEK5/TdT (B) mice. Mice were treated with tamoxifen daily for $7 \mathrm{~d}$ and perfused 3 weeks later. Scale bar, $10 \mu \mathrm{m}$. C, Quantification of TdT (+) and active caspase-3 $(+)$ cells in the $\mathrm{GCL}$ of the $0 \mathrm{~B} ; n=3$ mice/group; ${ }^{*} p<0.05$.

control mice in either cohort (Fig. 14). These data suggest that enhanced adult neurogenesis by ERK5 activation does not necessarily improve the retention of long-term olfactory memory.

\section{NesCreER:caMEK5 mice exhibit improved olfactory functional recovery after zinc-induced MOE lesion}

NesCreER:caMEK5 and vehicle control mice were given intranasal $\mathrm{ZnSO}_{4}$ irritation $(0.17 \mathrm{M}, 5 \mu \mathrm{l}$ per nostril $)$ to cause a lesion in the MOE, and the recovery of olfactory function was continuously monitored for 3 months. The $\mathrm{ZnSO}_{4}$ dosage used in this study, which is sufficient to generate a structural lesion in the MOE but still allowing a functional recovery, was optimized through a pilot study (data not shown). A habituation-dishabituation assay was used to assess mouse's ability to detect odors and discriminate between different odors before and after the MOE lesion.

Both NesCreER:caMEK5 and vehicle control mice demonstrated similar odor discrimination before $\mathrm{ZnSO}_{4}$ treatment (Fig. 15A). Six days after $\mathrm{ZnSO}_{4}$ treatment, neither groups showed any significant sniffing toward either citralva or vanillin (Fig. 15B), confirming that $\mathrm{ZnSO}_{4}$-induced MOE lesion caused a complete loss of olfactory function in both groups. The lack of any odor detection persisted at $21 \mathrm{~d}$ (Fig. 15C) and $35 \mathrm{~d}$ (Fig. 15D). However, 10 weeks after treatment, the NesCreER:caMEK5 mice demonstrated a significant recovery in odor detection and discrimination (Fig. 15E), which was confirmed at 12 weeks (Fig. 15F). In contrast, the vehicle control mice did not show any recovery in odor detection until 12 weeks postlesion.

To rule out the possibility that the enhanced recovery of olfactory function in NesCreER:caMEK5 mice is due to their altered mobility, we subjected both groups of mice to an open-field test to evaluate their general mobility. There was no significant difference in spontaneous movement between NesCreER:caMEK5 and vehicle control mice 12 weeks after $\mathrm{ZnSO}_{4}$ lesion (Fig. 15G). Additionally, both groups of mice achieved a similar level of body weight recovery and gain after MOE ablation (Fig. 15H). These data demonstrate that the enhanced recovery of olfactory functions in NesCreER: caMEK5 mice was not due to changes in basal locomotor activity or general health status of the mice.

The improved recovery of olfactory function observed in the NesCreER: caMEK 5 mice could be due to a better recovery of the olfactory sensory neurons in the MOE and/or enhanced adult neurogenesis in the OB. However, the expression of TdTomato reporter upon tamoxifen treatment was very limited in the MOE and was only seen in a few small blood vessels. TdTomato was not expressed in the olfactory sensory neurons, identified by olfactory marker protein (OMP), a specific marker for mature olfactory sensory neurons (Fig. 16A). Also, Nestin was not expressed in olfactory sensory neurons (Fig. 16B). These data suggest that the NesCreER:caMEK5-eGFP was not expressed in the MOE or to play a role in the recovery of olfactory sensory neurons in the MOE of NesCreER:caMEK5eGFP mice.

The $\mathrm{ZnSO}_{4}$ lesioned mice were directly evaluated for their olfactory sensory neurons recovery in the MOE right after the end of the behavioral studies. Both vehicle control and NesCreER: caMEK5 mice showed partial recovery in the olfactory epithelium after $\mathrm{ZnSO}_{4}$ lesion, demonstrated by Nissl staining and OMP expression (Fig. 16C-F). The extent of MOE recovery was comparable between the vehicle control and NesCreER:caMEK5 mice $(p<0.05)$.

Finally, to confirm that enhanced adult neurogenesis indeed occurred in the $\mathrm{OB}$ of NesCreER:caMEK5 mice after $\mathrm{ZnSO}_{4}$ lesion, we examined the adult-born interneurons in the $\mathrm{OB}$ after the end of the behavioral studies. Compared with the vehicle control mice, NesCreER:caMEK5 mice had significantly more newborn neurons in the OB (Fig. 16G,H). Together, these data suggest that enhanced adult neurogenesis in the OB likely contributes to the improved functional recovery of olfaction observed in the NesCreER:caMEK5 mice.

\section{Discussion}

In the adult brain, the olfactory bulb interneurons are continuously renewed throughout the life span. However, whether these adult-born neurons impact olfactory behavior is still uncertain. Furthermore, although adult-born neurons have been well characterized at the cellular level in the $\mathrm{OB}$, signaling mechanisms regulating adult $\mathrm{OB}$ neurogenesis are not fully delineated. As adult neurogenesis and olfactory function decline with aging or neurological disorders, there is considerable interest in developing strategies to promote adult olfactory neurogenesis 

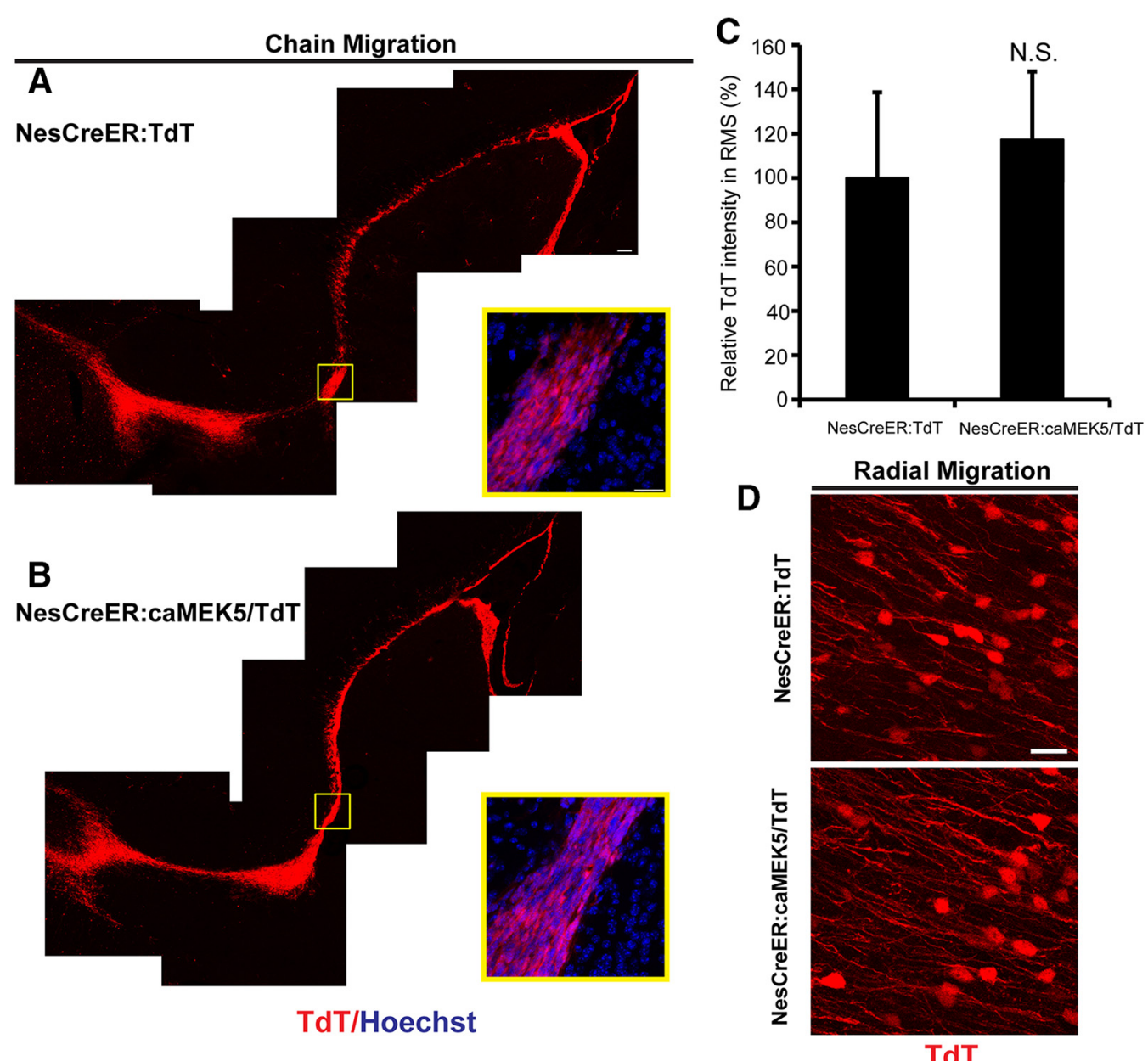

Figure 9. Inducible and conditional caMEK5 activation of ERK5 does not overtly affect chain migration in the RMS or radial migration in the 0B. NesCreER:caMEK5/TdT mice and NesCreER:TdT control littermates were treated with tamoxifen for $7 \mathrm{~d}$ and perfused $10 \mathrm{~d}$ after the last dose of tamoxifen. Tissue sections were immunostained for TdTomato. $A, B$, Representative images from sagittal sections show newborn cells from NesCreER:TdT control and NesCreER:caMEK5/TdT migrate similarly along the RMS. Scale bar, $100 \mu \mathrm{m}$; enlarged image, $25 \mu \mathrm{m}$. C, Quantification of TdTomato fluorescence intensity along the RMS. N.S., Not statistically significant. $\boldsymbol{D}$, Representative images from coronal sections show similar radial migration pattern in the $0 B$ of both mice. Scale bar, $25 \mu \mathrm{m} . n=3$ mice/group.

and improve functional olfactory recovery following lesions or disorders. We recently generated a line of knock-in mice where endogenous ERK5 in adult neural stem cells can be conditionally activated by tamoxifen-induced expression of a constitutive active MEK5 through Nestin-CreER ${ }^{\mathrm{TM}}$-mediated recombination (Wang et al., 2014). We reported that such conditional activation of endogenous ERK5 increases adult neurogenesis in the DG of the hippocampus and extends hippocampus-dependent memory (Wang et al., 2014). In this study, we used this caMEK5 knock-in mouse strain to determine whether conditional activation of endogenous ERK5 is sufficient to stimulate adult neurogenesis in the $\mathrm{OB}$, enhance olfactory function, and promote olfactory recovery after MOE lesioning.

In this report, we provide additional evidence that the expression of caMEK5-eGFP fusion protein is tightly controlled by Cremediated recombination in neural stem and progenitor cells both in vitro and in vivo, and when expressed, caMEK5-eGFP specifically activates endogenous ERK5 but not the closely related ERK1/2. In vitro studies using SVZ-aNPC cells cultured from caMEK5-eGFP ${ }^{\text {loxp/loxp }}$ mice showed that activation of endogenous ERK5 increases neuronal differentiation and promotes cell survival. Furthermore, by using two strains of knock-in mice, the
NesCreER:caMEK5 and NesCreER:caMEK5/TdT, we demonstrated that expression of caMEK5 increases the number of adultborn interneurons in the $\mathrm{OB}$ by promoting cell survival, neuronal differentiation, and maturation of adult-born cells without affecting cell proliferation, migration, or the stem-cell pool. Therefore, the inducible and targeted activation of ERK5 was sufficient to stimulate adult neurogenesis in the OB.

A number of studies have attempted to address the role of adult neurogenesis in olfactory behavior by suppressing adult neurogenesis (Gheusi et al., 2000; Lledo et al., 2006; Moreno et al., 2009; Lazarini and Lledo, 2011; Breton-Provencher and Saghatelyan, 2012; Pan et al., 2012a). Although these findings were not always consistent, several recent studies led to the exciting discovery that adult olfactory neurogenesis may impact several forms of olfactory behavior in mice (Gheusi et al., 2000; Lledo et al., 2006; Moreno et al., 2009; Breton-Provencher and Saghatelyan, 2012; Pan et al., 2012a). However, only a few reports have investigated whether olfactory function might be improved by increasing adult neurogenesis. For example, animals housed in an odorenriched environment for $40 \mathrm{~d}$ showed increased adult olfactory neurogenesis and improved olfactory memory (Rochefort et al., 2002), but the underlying signaling mechanisms remain unknown. Conditional deletion of Pten, a tumor suppressor gene, in 

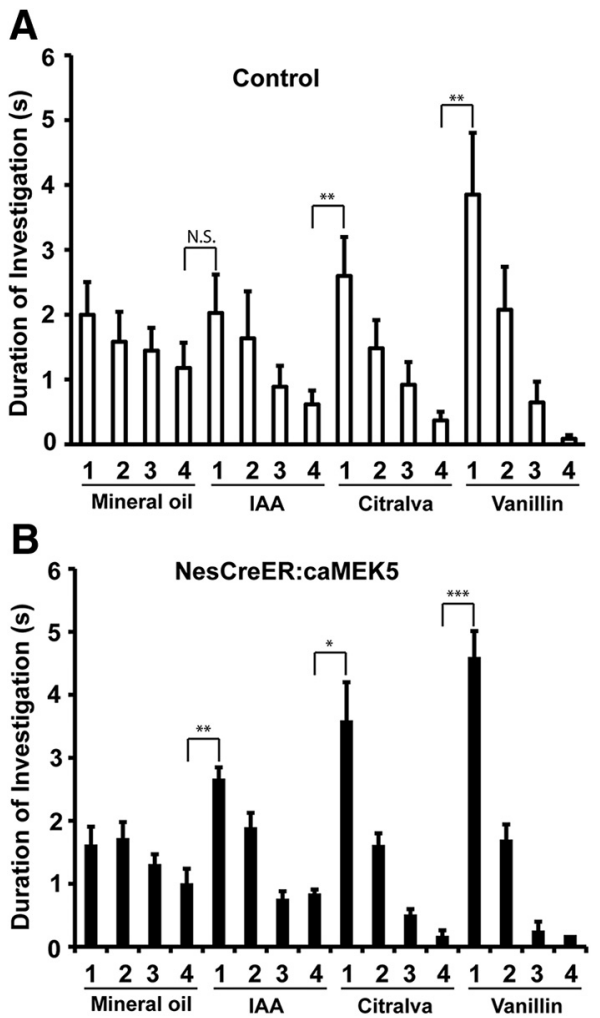

Figure 10. NesCreER:caMEK5 and control mice have comparable olfactory detection to discrete odorants in habituation/dishabituation assays. Control $(\boldsymbol{A})$ and NesCreER:caMEK5 mice ( $\boldsymbol{B})$ were pretrained with four presentations of mineral oil-soaked cotton swabs, then exposed to three odorants, IAA, citralva, and ethyl vanillin, each with four presentations. Stepwise decrease in the duration of investigations during sequential presentations of the same odor followed by renewed interest in investigation of the new odorant suggests normal olfactory habituation/ dishabituation; $n=9-10$ mice/group. N.S., Not statistically significant; ${ }^{*} p<0.05,{ }^{* *} p<$ $0.01,{ }^{* * *} p<0.001$.

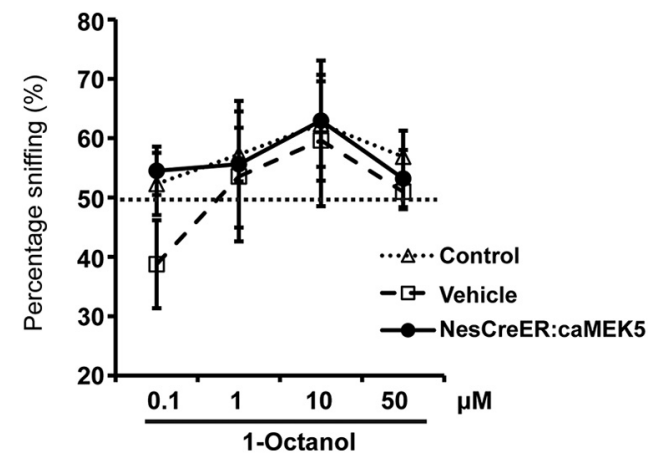

Figure 11. NesCreER:caMEK5 mice have normal odorant detection threshold. NesCreER: caMEK5, control, and vehicle mice were presented with two cotton swabs, one laced with mineral oil (vehicle) and the other with increasing concentrations of 1-octanol. An $>50 \%$ sniffing duration (above chance) indicates odorant detection; $n=9-10$ mice/group.

neural stem/progenitor cells driven by Cre expression under the control of a mouse GFAP promoter, enhances olfactory neurogenesis in the adult and facilitates $\mathrm{OB}$ recovery from acute damage (Gregorian et al., 2009). These studies are interesting and generated much excitement about the possibility that enhancing adult neurogenesis may improve olfactory function.

In this study, we use a more selective approach, the conditional and inducible activation of the ERK5 signaling pathway, to specifically stimulate adult neurogenesis. Data presented here
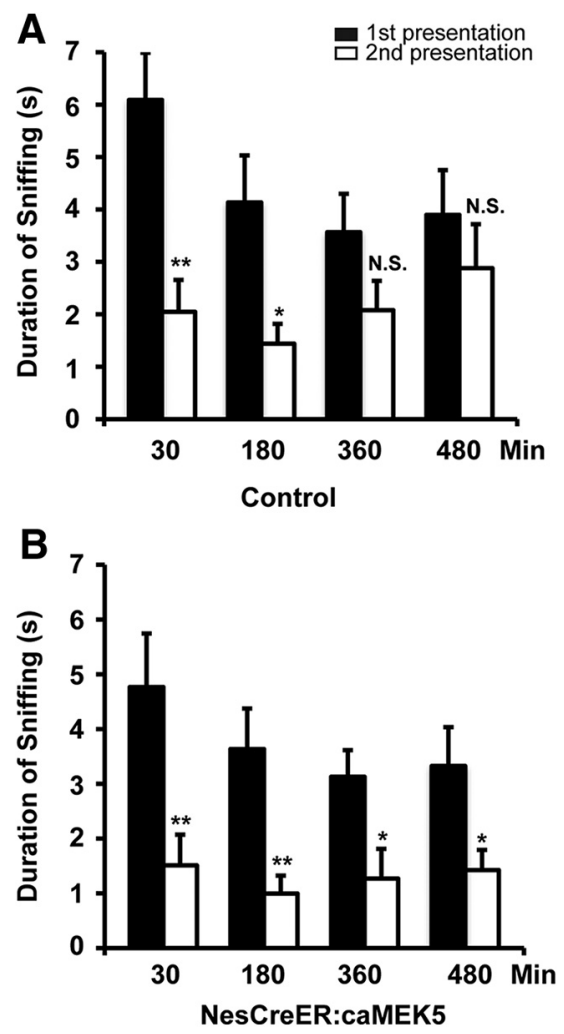

Figure 12. NesCreER:caMEK5 mice show enhanced olfactory short-term memory. NesCreER: caMEK5 and control mice were presented with cotton swabs laced with the same odorant twice at the indicated time intervals. A different odor was used for each interval time point and the sniffing duration of the cotton swab was recorded for both exposure sessions. A decrease in investigation during the second exposure of the same odorant suggests olfactory memory of the odorant. $\boldsymbol{A}$, Short-term memory test of the control mice. $\boldsymbol{B}$, Short-term memory test of the NesCreER:caMEK5 mice; $n=9-10$ mice/group; ${ }^{*} p<0.05,{ }^{* *} p<0.01$; N.S., not significant.

provide strong and clear genetic evidence supporting a causal relationship between enhanced adult neurogenesis and improved olfactory function. We found that ERK5 activation specifically in neurogenic regions of the adult mouse brain did not have a significant effect on olfactory discrimination in the habituation/ dishabituation assays or in the olfactory detection sensitivity. However, NesCreER:caMEK5 mice showed enhanced shortterm olfactory memory toward previously exposed odor compared with control littermates. This improved short-term memory phenotype is similar to that found in mice housed in odor-enriched environment (Rochefort et al., 2002). Together with recent reports that attenuated bulbar neurogenesis, either by anti-mitotic drugs (Breton-Provencher et al., 2009) or genetic approaches (Pan et al., 2012a), impairs short-term olfactory memory, these results suggest that ongoing adult olfactory neurogenesis is both necessary for and sufficient to improve shortterm olfactory memory.

We previously reported a novel role for adult neurogenesis in the acquisition of odor-cued associative learning (Pan et al., 2012a). Here we demonstrate that although both groups of mice learned the task of associating food reward with a specific odor, NesCreER:caMEK5 mice showed a better learning curve than control mice both in the regular training ( 8 trials/d for $8 \mathrm{~d}$ ) or more challenging training ( 4 trials/d for $10 \mathrm{~d}$ ) paradigms. These data provide the first evidence that enhanced adult neurogenesis can promote the acquisition of odor-cued associative learning, a process that is independent of the hippocampus (Kaut and Bun- 
sey, 2001; Kaut et al., 2003; Jonasson et al., 2004; Sultan et al., 2010).

Although NesCreER:caMEK5 mice demonstrated significantly better acquisition in odor-cued associative learning than controls, there was no concomitant increase in long-term memory retention compared with controls. This is in-line with recent reports that showed mice with ablations of adult bulbar neurogenesis with genetic (Imayoshi et al., 2008) or pharmacological (Breton-Provencher et al., 2009) approaches performed normally in olfactory long-term retention. These findings support the argument that shortterm olfactory memory is stored in the olfactory bulb, whereas long-term olfactory memory occurs in the cortical areas (Rochefort et al., 2002; Kelley et al., 2005).

Chemical ablation of the MOE destroys a mouse's ability to detect odorants. However, the MOE tissue does regenerate and the treated mice can recover some olfactory function. Interestingly, the $\mathrm{Ne}$ sCreER:caMEK5 mice regained ability to detect odorants sooner than control mice. We provide evidence that NesCreER: caMEK5-eGFP is not expressed in the MOE and that the recovery of olfactory sensory neurons does not significantly differ between the control and NesCreER: caMEK5 mice, ruling out the possibility that the improved recovery of olfaction function in the NesCreER:caMEK5 mice is due to a better MOE regeneration. However, deprivation of olfactory sensory input also causes cell death of adult-born neurons in the OB. We found that there are more newborn neurons $(\mathrm{NeuN}+$ and $\mathrm{BrdU}+$ ) in the OB of NesCreER:caMEK5 mice than in the control mice following MOE lesion and recovery. The enhanced neuronal differentiation and survival of the adult-born interneurons in the $\mathrm{OB}$ of NesCreER:caMEK5 mice may improve olfactory signal transduction from the olfactory epithelium to the brain, contributing to the improved olfactory recovery post-MOE lesion.

Despite recent exciting advances made in adult neurogenesis, underlying signaling mechanisms remain to be defined. We recently demonstrated that conditional knock-out of ERK5 in the adult neurogenic regions impairs neuronal differentiation, migration, and survival of adult-born neurons in the olfactory bulb (Li et al., 2013b). It also leads to deficits in several forms of olfactory behavior including reduced short-term olfactory memory and impaired acquisition of odorcued associative olfactory learning (Pan et al., 2012a). In this study, we provide evidence that conditional activation of ERK5 signaling in the adult neurogenic regions enhances neuronal dif-
Cohort 1

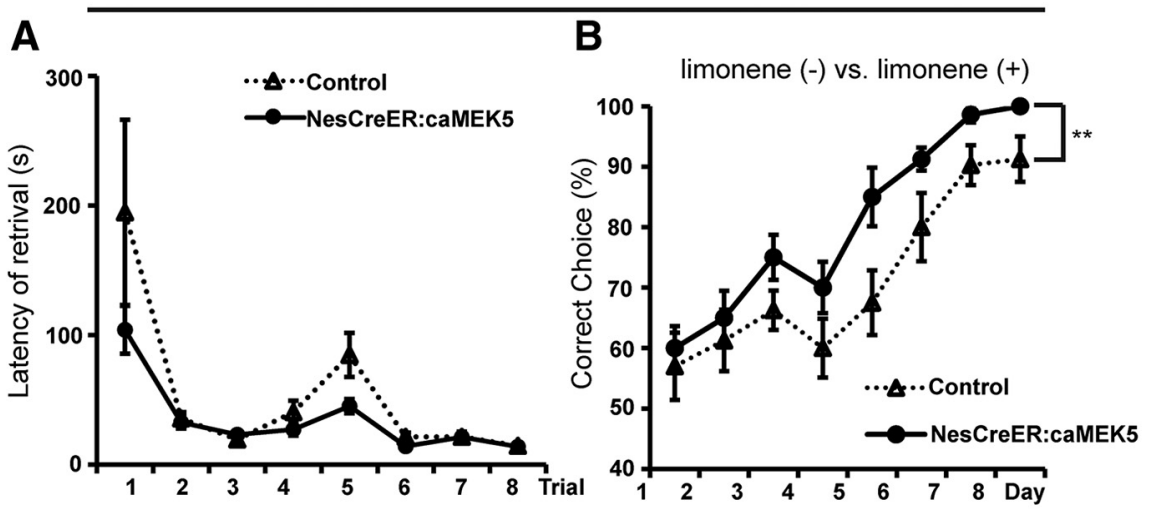

Cohort 2
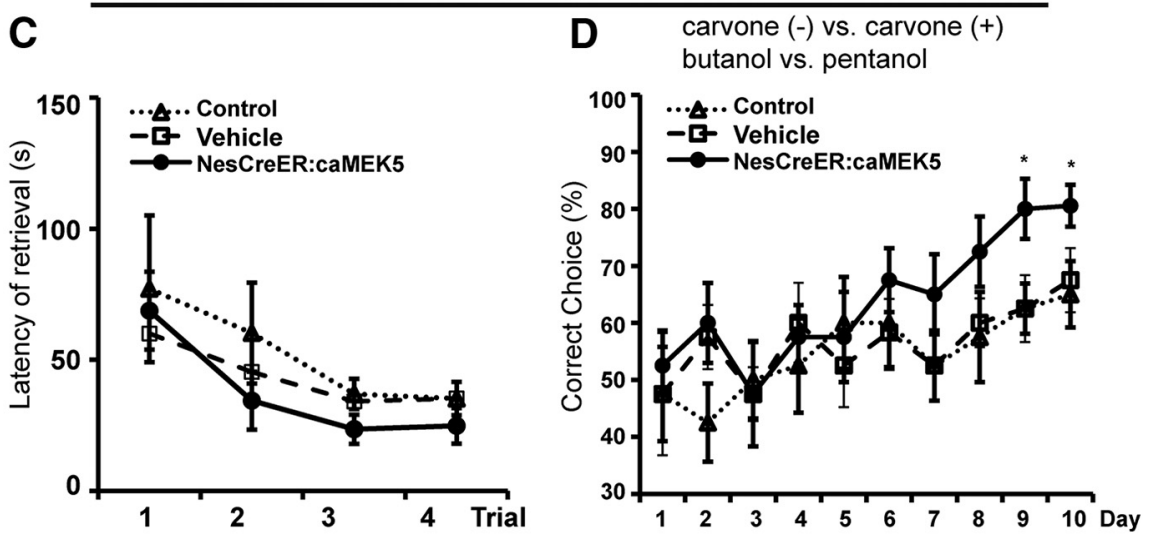

Figure 13. NesCreER:caMEK5 mice show better odor-cued associative olfactory learning. $A$, On the third day of pretraining, both control and NesCreER:caMEK5 mice learned to retrieve food reward that was deeply buried in the sand. $\boldsymbol{B}$, NesCreER:caMEK5 mice showed enhanced ability compared with control mice in associating food reward with limonene $(+)$ over an $8 \mathrm{~d}$ training course with eight trials per day; $n=9-10$ mice/group. $C, D, A$ different cohort of mice was assessed for odor-cued associative olfactory learning with a more challenging form of the sand-digging task. On the third day of pretraining, control, vehicle, and NesCreER:caMEK5 mice all learned to retrieve food reward that was deeply buried in the sand $(\boldsymbol{C}$. NesCreER:caMEK5 mice performed better than the two control groups in associating food reward with carvone $(+)$ and butanol (D); $n=9-10$ mice/group; ${ }^{*} p<0.05,{ }^{* *} p<0.01$.

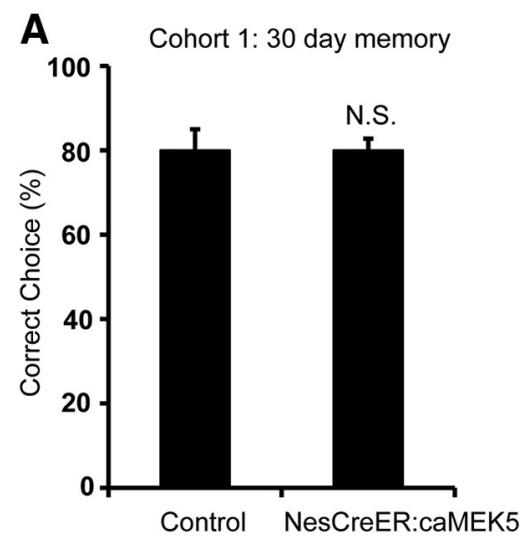

B Cohort 2: 21 day memory

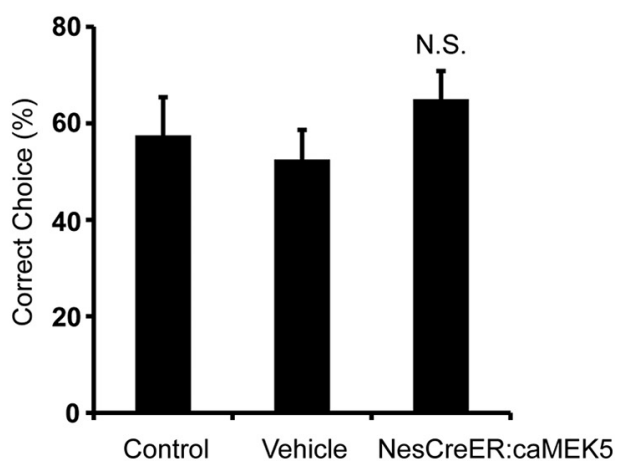

Figure 14. Nes(reER:caMEK5 mice have similar long-term olfactory memory as control mice. $A$, Thirty days after the mice finished the "standard" sand-digging task (Fig. 10B), mice (cohort 1) were tested for long-term olfactory memory. No significant difference in food reward retrieval was found between NesCreER:caMEK5 mice and control mice. $\boldsymbol{B}$, Three weeks after the mice finished the challenging form of associative learning task (Fig. 10D), mice (cohort2) were tested again for theirl long-term olfactorymemory. No significant differenceinfood reward retrieval was found between NesCreER:caMEK5 mice and the two control groups; $n=9-10$ mice/group. N.S., Not statistically significant. ferentiation and survival in the olfactory bulb, and improves short-term olfactory memory as well as acquisition of odor-cued associative olfactory learning. Thus, ERK5 activation seems to be both necessary and sufficient to promote adult neurogenesis in 
A
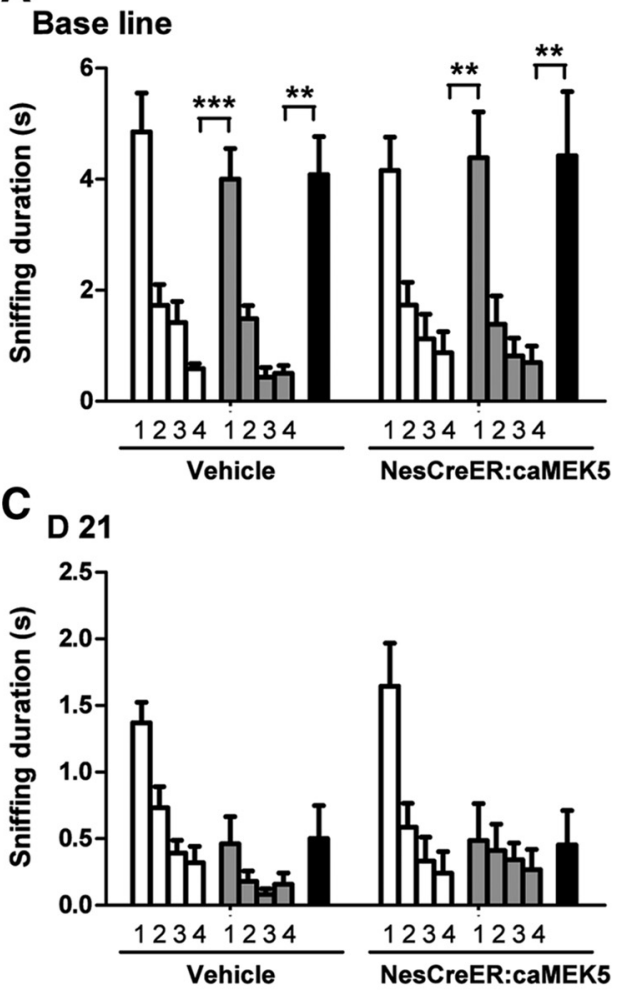

E D 70

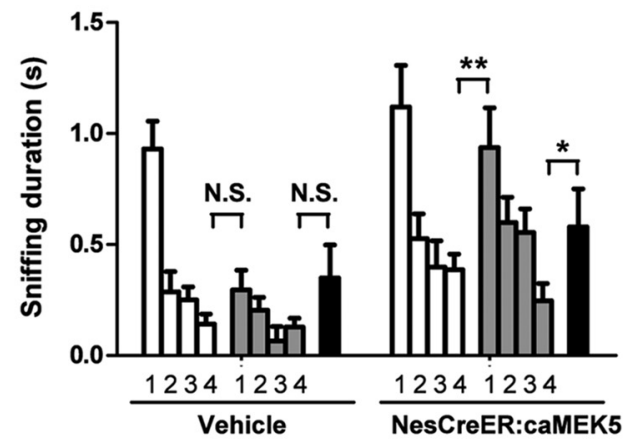

G

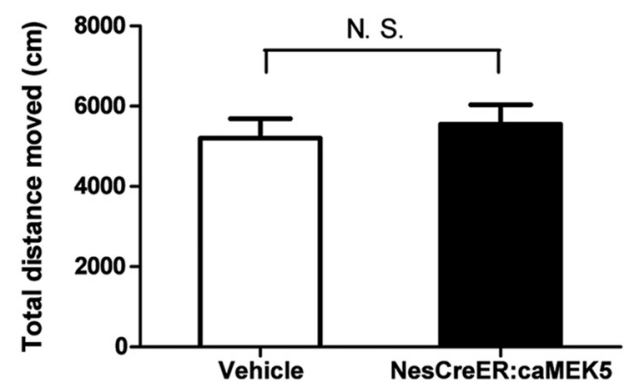

B
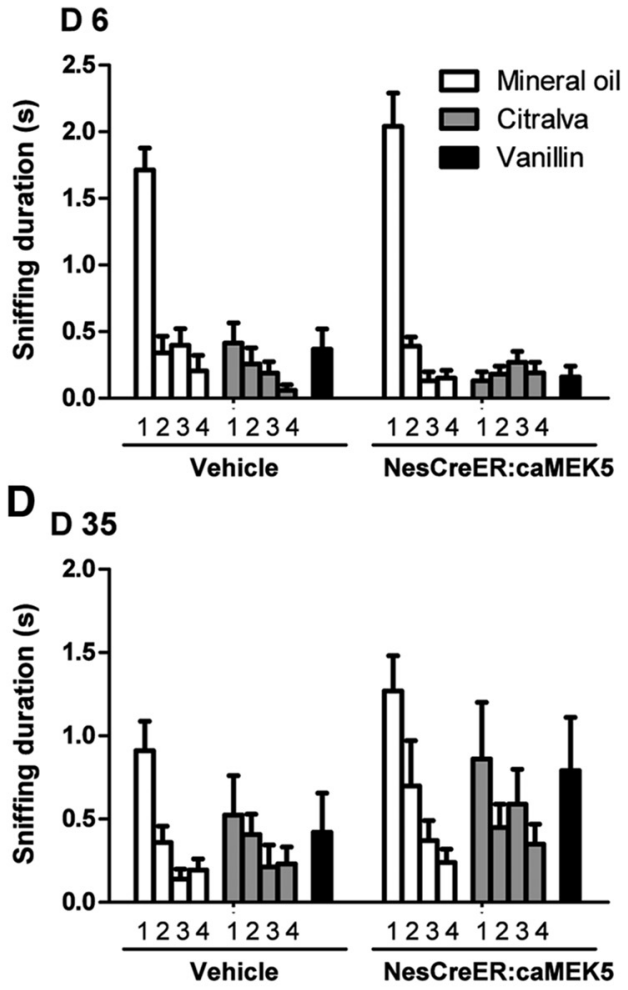

F D 84

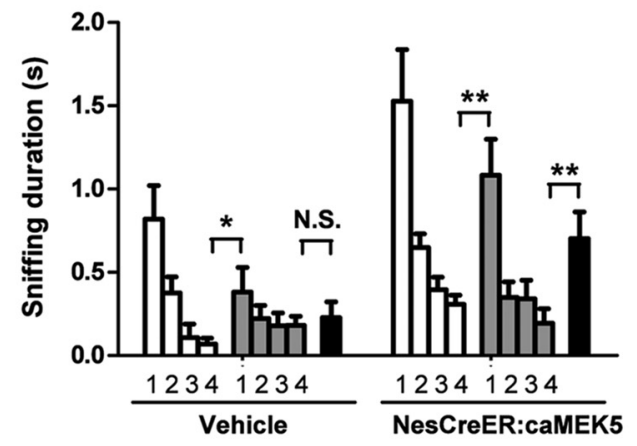

H

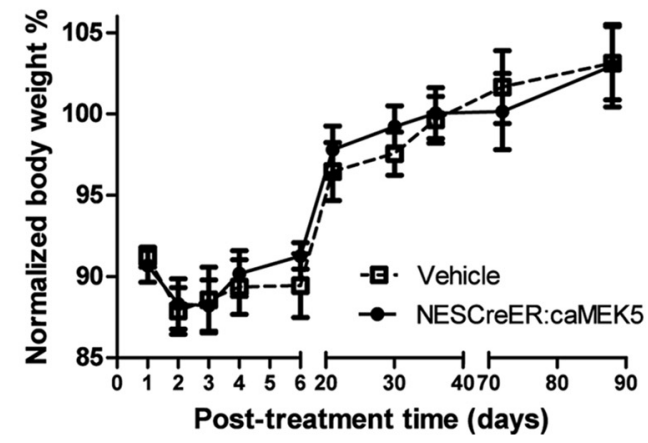

Figure 15. Olfactory function recovery after $\mathrm{ZnSO}_{4}$ treatment. $\boldsymbol{A}-\boldsymbol{F}$, Olfactory habituation-dishabituation assays at different time points: before $\mathrm{ZnSO}_{4}$ treatment (base line; $\boldsymbol{A}$ ), and $6 \mathrm{~d}$ $(\boldsymbol{B}), 21 \mathrm{~d}(\boldsymbol{C}), 35 \mathrm{~d}(\boldsymbol{D}), 70 \mathrm{~d}(\boldsymbol{E})$, and $84 \mathrm{~d}(\boldsymbol{F})$ after $\mathrm{ZnSO}_{4}$ treatment. Comparison of sniffing time was made between the first presentation of a novel odor and the last presentation of the previous odor. $\mathbf{G}$, Total distance moved in a 30 min open-field test at $84 \mathrm{~d}$ after $\mathrm{ZnSO}_{4}$ treatment. $\boldsymbol{H}$, Relative body weight gain over the course of recovery from $\mathrm{ZnSO}_{4}$-induced lesion. Values are presented as a percentage normalized to the individuals' body weight before $\mathrm{ZnSO}_{4}$ treatment; $n=7-9 /$ group; N.S., not statistically significant; ${ }^{*} p<0.05$, ${ }^{* *} p<0.01$, ${ }^{* * *} p<0.001$. 

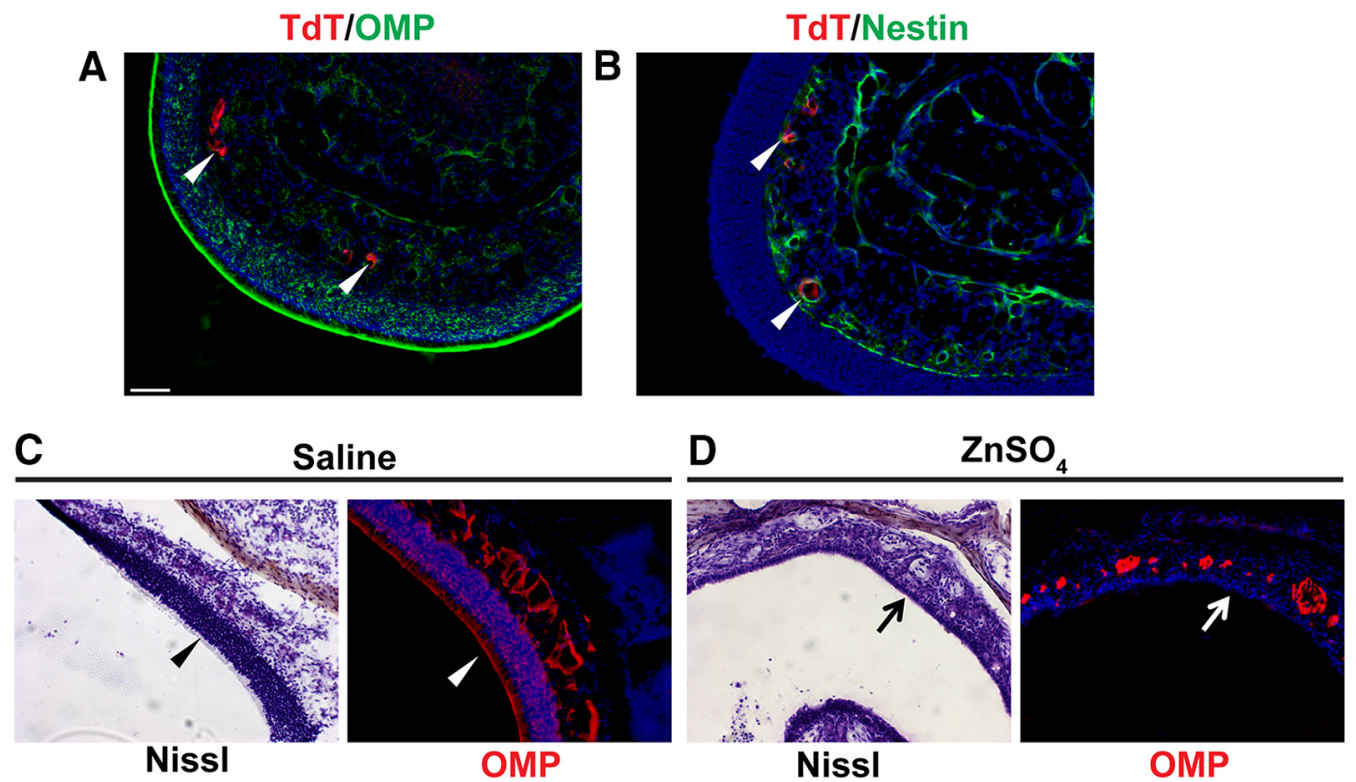

E

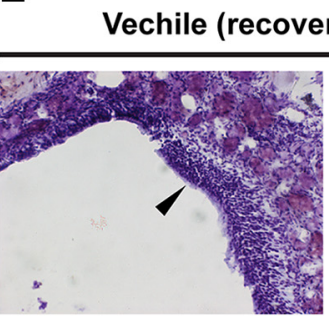

NissI

G

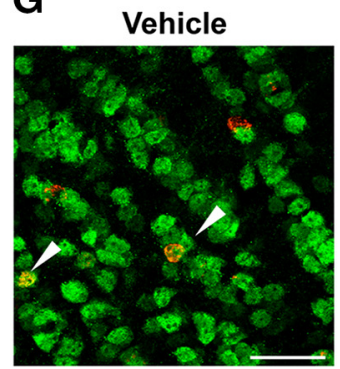

BrdU/NeuN
F

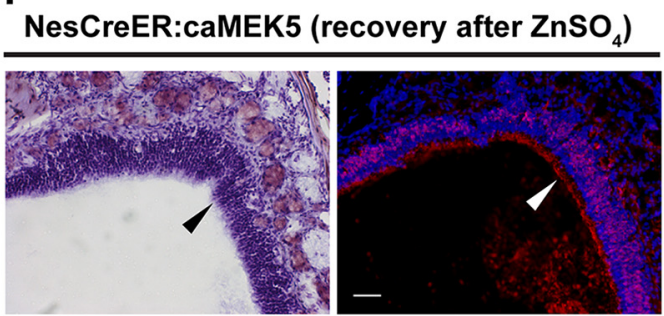

NissI

OMP

H

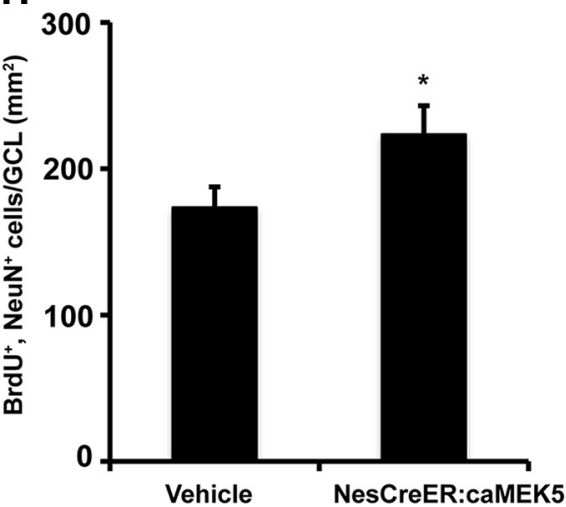

Figure 16. Improved olfactory functional recovery observed in the NesCreER:caMEK5 mice is likely due to enhanced neurogenesis in the OB. A, Representative images showing lack of coexpression of TdTomato (red) and OMP (green) in the MOE of NesCreER:TdT mice. Arrowheads indicate TdT ( + ) cells. B, Representative images showing expression of TdTomato (red) and Nestin (green) in the MOE of NesCreER:TdT mice. Arrowheads indicate the few TdT (+)/Nestin $(+)$ costaining in small, circular blood vessles. C, D, Nissl staining and OMP (red) expression in the MOE of the saline treated mice $(\boldsymbol{C})$ and $\mathrm{ZnSO}_{4}$-treated mice $(\boldsymbol{D})$. Mice were perfused $5 \mathrm{~d}$ post-treatment. Arrowheads point to the apical surface of olfactory epithelium, whereas arrows indicate $\mathrm{MOE}$ lesion. $\boldsymbol{E}$, $\boldsymbol{F}$, Representative images of Nissl staining and OMP (red) expression in the MOE of vehicle control mice $(\boldsymbol{E})$ and NesCreER:caMEK5 mice $(\boldsymbol{F}) 15$ weeks after $\mathrm{ZnSO}_{4}$ treatment. Arrowheads point to recovered MOE. Scale bars: $\boldsymbol{A}-\boldsymbol{F}, 50 \mu \mathrm{m} . \boldsymbol{G}$, Representative confocal images of BrdU (red) and NeuN (green) in the OB of vehicle and NesCreER:caMEK5 mice 15 weeks after ZnSO treatment. Scale bars, $25 \mu \mathrm{m}$. $\boldsymbol{H}$, Quantification of BrdU ${ }^{+}$and NeuN ${ }^{+}$double-immunoreactive cells in the $\mathrm{GCL}$ of each OB 15 weeks after $\mathrm{ZnSO}_{4}$ treatment; $n=4-5$ mice/group; ${ }^{*} p<0.05$.

vivo, as well as to maintain and improve several forms of olfactory function under normal physiological conditions. Results from these two complimentary genetic approaches establish a unified theme implicating ERK5 as a key regulator of adult neurogenesis in the OB.

MEK5-ERK5 is an endogenous MAP kinase signaling pathway that can be activated by extracellular signals present in the SVZ that are known to promote adult neurogenesis, including neurotrophins and prolactin (Li et al., 2013a; Wang et al., 2013).
Our data provides the first evidence that direct activation of a single endogenous signaling pathway, the ERK5 pathway, selectively in adult neurogenic regions, is sufficient to enhance adult neurogenesis in the SVZ/OB and improve short-term olfactory memory, odor-cued associative learning, and olfaction recovery after lesion. The majority of the adult-born cells in the SVZ do not survive under normal physiological conditions, and the failure rate may be even higher in injury or disease conditions (Turnley et al., 2014). We propose that activation of the endogenous 
ERK5 signaling pathway may enhance survival and neuronal differentiation following injury.

\section{References}

Breton-Provencher V, Saghatelyan A (2012) Newborn neurons in the adult olfactory bulb: unique properties for specific odor behavior. Behav Brain Res 227:480-489. CrossRef Medline

Breton-Provencher V, Lemasson M, Peralta MR 3rd, Saghatelyan A (2009) Interneurons produced in adulthood are required for the normal functioning of the olfactory bulb network and for the execution of selected olfactory behaviors. J Neurosci 29:15245-15257. CrossRef Medline

Cavanaugh JE, Ham J, Hetman M, Poser S, Yan C, Xia Z (2001) Differential regulation of mitogen-activated protein kinases ERK1/2 and ERK5 by neurotrophins, neuronal activity, and cAMP in neurons. J Neurosci 21: 434-443. Medline

Dunston D, Ashby S, Krosnowski K, Ogura T, Lin W (2013) An effective manual deboning method to prepare intact mouse nasal tissue with preserved anatomical organization. J Vis Exp 78:e50538. CrossRef Medline

English JM, Vanderbilt CA, Xu S, Marcus S, Cobb MH (1995) Isolation of MEK5 and differential expression of alternatively spliced forms. J Biol Chem 270:28897-28902. CrossRef Medline

Gheusi G, Cremer H, McLean H, Chazal G, Vincent JD, Lledo PM (2000) Importance of newly generated neurons in the adult olfactory bulb for odor discrimination. Proc Natl Acad Sci U S A 97:1823-1828. CrossRef Medline

Gregorian C, Nakashima J, Le Belle J, Ohab J, Kim R, Liu A, Smith KB, Groszer M, Garcia AD, Sofroniew MV, Carmichael ST, Kornblum HI, Liu $\mathrm{X}, \mathrm{Wu} \mathrm{H}$ (2009) Pten deletion in adult neural stem/progenitor cells enhances constitutive neurogenesis. J Neurosci 29:1874-1886. CrossRef Medline

Imayoshi I, Sakamoto M, Ohtsuka T, Takao K, Miyakawa T, Yamaguchi M, Mori K, Ikeda T, Itohara S, Kageyama R (2008) Roles of continuous neurogenesis in the structural and functional integrity of the adult forebrain. Nat Neurosci 11:1153-1161. CrossRef Medline

Jonasson Z, Ballantyne JK, Baxter MG (2004) Preserved anterograde and retrograde memory of rapidly acquired olfactory discriminations after neurotoxic hippocampal lesions. Hippocampus 14:28-39. CrossRef Medline

Kageyama R, Imayoshi I, Sakamoto M (2012) The role of neurogenesis in olfaction-dependent behaviors. Behav Brain Res 227:459-463. CrossRef Medline

Kaut KP, Bunsey MD (2001) The effects of lesions to the rat hippocampus or rhinal cortex on olfactory and spatial memory: retrograde and anterograde findings. Cogn Affect Behav Neurosci 1:270-286. CrossRef Medline

Kaut KP, Bunsey MD, Riccio DC (2003) Olfactory learning and memory impairments following lesions to the hippocampus and perirhinalentorhinal cortex. Behav Neurosci 117:304-319. CrossRef Medline

Kelley AE, Schiltz CA, Landry CF (2005) Neural systems recruited by drugand food-related cues: studies of gene activation in corticolimbic regions. Physiol Behav 86:11-14. CrossRef Medline

Kuo CT, Mirzadeh Z, Soriano-Navarro M, Rasin M, Wang D, Shen J, Sestan N, Garcia-Verdugo J, Alvarez-Buylla A, Jan LY, Jan YN (2006) Postnatal deletion of numb/numblike reveals repair and remodeling capacity in the subventricular neurogenic niche. Cell 127:1253-1264. CrossRef Medline

Lagace DC, Whitman MC, Noonan MA, Ables JL, DeCarolis NA, Arguello AA, Donovan MH, Fischer SJ, Farnbauch LA, Beech RD, DiLeone RJ, Greer CA, Mandyam CD, Eisch AJ (2007) Dynamic contribution of nestin-expressing stem cells to adult neurogenesis. J Neurosci 27:1262312629. CrossRef Medline

Lazarini F, Lledo PM (2011) Is adult neurogenesis essential for olfaction? Trends Neurosci 34:20-30. CrossRef Medline

Li T, Wang W, Pan YW, Xu L, Xia Z (2013a) A hydroxylated metabolite of flame-retardant PBDE-47 decreases the survival, proliferation and neu- ronal differentiation of primary cultured adult neural stem cells and interferes with signaling of ERK5 MAP kinase and neurotrophin 3. Toxicol Sci 134:111-124. CrossRef Medline

Li T, Pan YW, Wang W, Abel G, Zou J, Xu L, Storm DR, Xia Z (2013b) Targeted deletion of the ERK5 MAP kinase impairs neuronal differentiation, migration, and survival during adult neurogenesis in the olfactory bulb. PLoS One 8:e61948. CrossRef Medline

Lledo PM, Alonso M, Grubb MS (2006) Adult neurogenesis and functional plasticity in neuronal circuits. Nat Rev Neurosci 7:179-193. CrossRef Medline

Madisen L, Zwingman TA, Sunkin SM, Oh SW, Zariwala HA, Gu H, Ng LL, Palmiter RD, Hawrylycz MJ, Jones AR, Lein ES, Zeng H (2010) A robust and high-throughput Cre reporting and characterization system for the whole mouse brain. Nat Neurosci 13:133-140. CrossRef Medline

Moreno MM, Linster C, Escanilla O, Sacquet J, Didier A, Mandairon N (2009) Olfactory perceptual learning requires adult neurogenesis. Proc Natl Acad Sci U S A 106:17980-17985. CrossRef Medline

Pan YW, Kuo CT, Storm DR, Xia Z (2012a) Inducible and targeted deletion of the ERK5 MAP kinase in adult neurogenic regions impairs adult neurogenesis in the olfactory bulb and several forms of olfactory behavior. PLoS One 7:e49622. CrossRef Medline

Pan YW, Chan GC, Kuo CT, Storm DR, Xia Z (2012b) Inhibition of adult neurogenesis by inducible and targeted deletion of ERK5 mitogenactivated protein kinase specifically in adult neurogenic regions impairs contextual fear extinction and remote fear memory. J Neurosci 32:64446455. CrossRef Medline

Pan YW, Zou J, Wang W, Sakagami H, Garelick MG, Abel G, Kuo CT, Storm DR, Xia Z (2012c) Inducible and conditional deletion of extracellular signal-regulated kinase 5 disrupts adult hippocampal neurogenesis. J Biol Chem 287:23306-23317. CrossRef Medline

Pan YW, Wang W, Xia Z (2013a) Assessment of adult neurogenesis in mice. Curr Protoc Toxicol 12:Unit12.20. CrossRef Medline

Pan YW, Storm DR, Xia Z (2013b) Role of adult neurogenesis in hippocampus-dependent memory, contextual fear extinction and remote contextual memory: new insights from ERK5 MAP kinase. Neurobiol Learn Mem 105:81-92. CrossRef Medline

Rochefort C, Gheusi G, Vincent JD, Lledo PM (2002) Enriched odor exposure increases the number of newborn neurons in the adult olfactory bulb and improves odor memory. J Neurosci 22:2679-2689. Medline

Sultan S, Mandairon N, Kermen F, Garcia S, Sacquet J, Didier A (2010) Learning-dependent neurogenesis in the olfactory bulb determines longterm olfactory memory. FASEB J 24:2355-2363. CrossRef Medline

Turnley AM, Basrai HS, Christie KJ (2014) Is integration and survival of newborn neurons the bottleneck for effective neural repair by endogenous neural precursor cells? Front Neurosci 8:29. CrossRef Medline

Wang W, Pan YW, Wietecha T, Zou J, Abel GM, Kuo CT, Xia Z (2013) Extracellular signal-regulated kinase 5 (ERK5) mediates prolactinstimulated adult neurogenesis in the subventricular zone and olfactory bulb. J Biol Chem 288:2623-2631. CrossRef Medline

Wang W, Pan YW, Zou J, Li T, Abel GM, Palmiter RD, Storm DR, Xia Z (2014) Genetic activation of ERK5 MAP kinase enhances adult neurogenesis and extends hippocampus-dependent long-term memory. J Neurosci 34:2130-2147. CrossRef Medline

Whitman MC, Greer CA (2009) Adult neurogenesis and the olfactory system. Prog Neurobiol 89:162-175. CrossRef Medline

Zhou G, Bao ZQ, Dixon JE (1995) Components of a new human protein kinase signal transduction pathway. J Biol Chem 270:12665-12669. CrossRef Medline

Zou J, Pan YW, Wang Z, Chang SY, Wang W, Wang X, Tournier C, Storm DR, Xia Z (2012) Targeted deletion of ERK5 MAP kinase in the developing nervous system impairs development of GABAergic interneurons in the main olfactory bulb and behavioral discrimination between structurally similar odorants. J Neurosci 32:4118-4132. CrossRef Medline 